DFF $1 / 11 / 97$

\title{
Exact Results in Chern-Simons Supergravity
}

\author{
P. Valtancoli \\ Dipartimento di Fisica dell' Universita', Firenze \\ and INFN, Sezione di Firenze (Italy)
}

\begin{abstract}
We propose an extension of a recent non-perturbative method suited for solving the $N$-body problem in $(2+1)$-gravity to the case of Chern-Simons supergravity. Coupling with supersymmetric point particles is obtained implicitly by extending the $D J H$ matching conditions of gravity.

The consistent solution of the interacting case is obtained by building a general non-trivial mapping, extending the superanalytic mapping, between a flat polydromic $X^{M}$ supercoordinate system and a physical one $x^{N}$, representing the $D J H$ matching conditions around the superparticles. We show how to construct such a mapping in terms of analytic functions, and we give their exact expressions for the two body case. The extension to the $N$ body case is also discussed.

In the Minkoskian coordinates the superparticles move freely, and in particular the fermionic coordinates $\Theta\left(\xi_{(i)}^{N}\right)$ are constants, whose values can be fixed by using the monodromy properties. While the bosonic part of the supergeodesic equations are obtained, as in gravity, by measuring the bosonic distance in Minkowskian space-time, we find that the fermionic geodesic equations can be defined only by requiring that a non-perturbative divergence of the $X^{M}=X^{M}\left(x^{N}\right)$ mapping cancels out on the world-lines of the superparticles.
\end{abstract}




\section{Introduction}

Recently, a series of paper have renewed a considerable interest for the study of the classical problem of $N$ particles interacting with $(2+1)$-gravity [1]-[2]-[3]-[4]-[5]-[6]- [7]-[8]. In this paper we have investigated the supergravity case, following also ref. [9], applying an improved version of the method which enabled us to solve the classical $N$-body problem in $(2+1)$ gravity [7]. The motivation for such study is twofold, since firstly it gives an opportunity to study non-perturbatively for the first time a classical interacting solution in a supergravity theory, and secondly since it permits to study the spin as an anticommuting variable, already built in in the superalgebra. In fact this interest came to us after the study of spinning particles [10]- [1], which are affected by the $C T C$ problem [12]. In that case we found that closed temporal curves appear inside a $C T C$ horizon, surrounding the particle site, whose radius is of the order of $S^{2}$, the square of the spin. So what happens when instead of treating the spin as a classical variable we introduce it as a pseudoclassical variable ? The natural framework in which this idea could be tested is $(2+1)$ supergravity ( see [13 for a review ), where we are forced to write equations in the framework of pseudoclassical dynamics [14.

The particular problem of $N$ superparticles coupled to supergravity [9] seems particular interesting because captures many of the simplifying features of $(2+1)$ gravity. Let us briefly recall them: the space-time of a point source is a cone with deficit angle and can be represented as a Minkoskian space-time with an excised region [2]. Moreover the problem of finding the metric of $N$ bodies can be reduced to find a mapping between a multivalued Minkowskian coordinate system $X^{a}$ and a physical coordinate system $X^{a}=X^{a}\left(x^{b}\right)$ [5]. In a particular instantaneous gauge it was found in ref. [7] that such a Minkowskian coordinate system can be represented as a sum of polydromic analytic and antianalytic functions, with branch points at the particle sites. Then integrating the geodesic equations was found to be equivalent to measure the bosonic distance $X^{a}\left(\xi_{i}\right)-X^{a}\left(\xi_{j}\right)$ between the particles in the Minkowskian coordinates.

Since the same simplifications that permit to solve $(2+1)$-gravity still hold in the case of $(2+1)$-supergravity, we were encouraged to understand the quite involved equations of supergravity [13]-15 as a non-trivial, polydromic mapping between supercoordinates $X^{M}=X^{M}\left(x^{N}\right)$, where now the index $N$ covers also the anticommuting variables [16]-17]. As a first step we find that the simplest way to couple a superparticle to supergravity is to introduce a composite monodromy matrix, carrying not only Lorentz cuts but also supersymmetric cuts. In particular, in addition to the bosonic constants of motion, which appear as parameters into the definition of the monodromy matrix, we introduce fermionic constant parameters, induced by the supersymmetric source, which are useful to parameterize 
the solution for the gravitino field. These constants describe also the free motion of the superparticle in the flat superspace $X^{M}$, and in particular the fermionic coordinate $\Theta\left(\xi^{N}\right)$ of the superparticle is constant, whose value is determined by the monodromy matrix.

Then we are able to show that the polydromic supercoordinate transformation $X^{M}=$ $X^{M}\left(x^{N}\right)$ can be represented as a sum of analytic and anti-analytic functions, as in the case of gravity [7], whose form resembles the superanalytic mapping. The bosonic part of this mapping is simply dictated by the solution of the corresponding problem in $(2+1)$-gravity. The non-triviality of supergravity is hidden in finding a solution to a polydromic mapping for the gravitino field, which however can be solved exactly for the two body problem.

To explain our results on the superspace mapping it is better to review the results of gravity, which represents the pure bosonic part of it, if we avoid all the dependence from the anticommuting variables. In the case of massive spinless particles coupled to gravity the polydromy is well defined at the particle site, since it can be reduced to a pure rotation. In the case of spinning particles, the definition of such a mapping is more problematic, due to the translation monodromy giving rise, for a static particle, to a logarithmic cut in the $T$ variable. Therefore the $X^{a}$ variables are no more single valued at the particles sites, as noted in [5].

Moreover, for a static spinning source it was surprisingly found another non-perturbative divergence of the $Z$-mapping [8]-[11], apparently without any motivation although in our instantaneous gauge it is related to the $C T C$ problem [12]. In the framework of supergravity, we find that the $X^{M}=X^{M}\left(x^{N}\right)$ mapping is plagued with the same divergences of the spinning case, at least with our gauge choice. It appears a logarithmic divergence due to the spin, and again the same type of divergence analogous to the $Z$-mapping divergence of the spinning case. However, in this case the superspace geometry helps in giving an interesting interpretation to it. According to our computations, we find that the $\theta$ terms carry an analogous divergence, and that it is possible to cancel the fermionic residue of such divergence. Requiring such cancellation at the superparticle site is then equivalent to integrate the fermionic part of the supergeodesics equations in the present formalism, as measuring the bosonic distance between two particles in Minkowskian coordinates was equivalent to integrate the geodesic equations in gravity.

Therefore to study superparticle dynamics it is not possible to truncate the supermapping to the $\theta=0$ part. We also give a general proof that the mechanism of cancellation of divergences is self-consistent at all orders, and we determine the general solution for the fermionic geodesic equation. Finally we are also able to integrate the bosonic trajectories of the superparticles and give the classical scattering angle. 
The contents of the paper are as follows. In the introductory Section 2 we recall the Chern-Simons formalism for introducing $(2+1)$-supergravity and, following [7], we define the mapping problem from a multivalued flat supercoordinate system to single valued coordinates in a instantaneous gauge. In Sec. 3 we treat the two superbody problem, and in particular, we give the non perturbative interacting solution for the $X^{M}=X^{M}\left(x^{N}\right)$ mapping and the motion of the superparticles. We also outline how to solve the many body case. Finally we discuss our results in Section 4, giving a few concluding remarks.

\section{Supergravity Lagrangian}

The $N=1$ Super-Poincarè algebra $I S O(2,1 \mid 2)$ is given in terms of real generators as follows

$$
\begin{aligned}
& {\left[J^{a}, J^{b}\right]=\epsilon^{a b c} J_{c} \quad\left[J^{a}, Q_{\alpha}\right]=-\frac{1}{2} \gamma_{, \alpha}^{a \beta} Q_{\beta}} \\
& {\left[J^{a}, P^{b}\right]=\epsilon^{a b c} P_{c} \quad\left\{Q_{\alpha}, Q_{\beta}\right\}=-\frac{1}{4}\left(\gamma^{a} C^{-1}\right)_{\alpha \beta} P_{a}} \\
& {\left[P^{a}, P^{b}\right]=\left[P^{a}, Q_{\alpha}\right]=0,}
\end{aligned}
$$

where $C^{\alpha \beta}$ is the charge conjugation matrix $C^{12}=-C^{21}=1$ and $J^{a}=\frac{1}{2} \epsilon^{a b c} J_{b c}$.

Following Witten [18], we recall how to formulate $(2+1)$ gravity theories in terms of a topological Chern-Simons action. In general it can be written for a compact gauge group $G$ as

$$
I_{C S}=\frac{1}{2} \int_{M} \operatorname{Tr}\left(A \wedge d A+\frac{2}{3} A \wedge A \wedge A\right),
$$

with $A=A^{a} T_{a}$ a Lie-algebra-valued one form. The kinetic term is dependent on the choice of the trace operation. For compact Lie groups this is usually chosen as $d_{a b}=\operatorname{Tr}\left(T_{a} T_{b}\right)$. More generally, $d_{a b}$ is an ad-invariant quadratic form on the Lie algebra. To write a consistent action we must require that this quadratic form is non-degenerate. For the general case of the group $I S O(d-1,1)$, which is non semi-simple, there is no candidate for $d_{a b}$ because the natural quadratic Casimir $C_{1}=P^{a} P_{a}$ is degenerate. In the special $d=3$ case however there is another quadratic Casimir $C_{2}=J^{a} P_{a}$ which induces a natural non-degenerate quadratic form

$$
<J_{a}, P_{b}>=\delta_{a b}, \quad<J_{a}, J_{b}>=<P_{a}, P_{b}>=0 .
$$

Then the $I S O(2,1)$ action, endowed with the bilinear form (2.3), corresponds to the action of $(2+1)$-gravity. 
What happens in $(2+1)$ supergravity? The supersymmetric generators do not contribute to the mass Casimir $C_{1}=P_{a} P^{a}$, while the second Casimir is modified as

$$
C_{2}=P_{a} J^{a}+\bar{Q}^{\alpha} Q_{\alpha}
$$

where $\bar{Q}^{\alpha}=Q_{\beta} C^{\beta \alpha}$. It induces a corresponding quadratic form $d^{A B}$, with which it is possible to write down the supergravity Lagrangian as a Chern-Simons theory on the $N=1$ SuperPoincarè algebra $I S O(2,1 \mid 2)$. Defining

$$
A_{\mu}=e_{\mu}^{a} P_{a}+\omega_{\mu}^{a} J_{a}+\bar{\psi}_{\mu}^{\alpha} Q_{\alpha},
$$

the Chern-Simons action for $N=1$ supergravity is defined by (2.2). From $d_{A B}$ defined from (2.4), we obtain the usual action for simple supergravity

$$
\begin{aligned}
\mathcal{L} & =-\frac{1}{2} \int_{M} d^{3} x \epsilon^{\mu \nu \rho}\left[e_{\mu}^{a}\left(\partial_{\nu} \omega_{a \rho}-\partial_{\rho} \omega_{a \nu}+\epsilon_{a b c} \omega_{\nu}^{b} \omega_{\rho}^{c}\right)+\right. \\
& \left.+\frac{1}{2} \bar{\psi}_{\mu}\left(\partial_{\nu}+\frac{1}{2} \omega_{\nu}^{a} \gamma_{a}\right) \psi_{\rho}\right] .
\end{aligned}
$$

The equations of motion of $(2+1)$-supergravity reveal the presence of a distributed torsion source, due to the presence of the gravitino field

$$
\begin{aligned}
\partial_{\mu} \omega_{a \nu} & -\partial_{\nu} \omega_{a \mu}+\epsilon_{a b c} \omega_{\mu}^{b} \omega_{\nu}^{c}=0 \\
\partial_{\mu} e_{a \nu} & -\partial_{\nu} e_{a \mu}+2 \epsilon_{a b c} e_{\mu}^{b} \omega_{\nu}^{c}+\frac{1}{8} \bar{\psi}_{\mu} \gamma_{a} \psi_{\nu}-\frac{1}{8} \bar{\psi}_{\nu} \gamma_{a} \psi_{\mu}=0 \\
\left(\partial_{\mu}\right. & \left.+\frac{1}{2} \omega_{\mu}^{a} \gamma_{a}\right) \psi_{\nu}-\left(\partial_{\nu}+\frac{1}{2} \omega_{\nu}^{a} \gamma_{a}\right) \psi_{\mu}=0
\end{aligned}
$$

These can be simplified by going to a singular gauge, similar to what has been discussed in [5] for gravity, and choosing a polydromic dreibein and gravitino fields such that $\omega_{a \mu}=0$ locally, from which one obtains the simplified equations:

$$
\begin{aligned}
\omega_{\mu}^{a} & =0 \\
\partial_{\mu} e_{\nu}^{a} & -\partial_{\nu} e_{\mu}^{a}+\frac{1}{8} \bar{\psi}_{\mu} \gamma^{a} \psi_{\nu}-\frac{1}{8} \bar{\psi}_{\nu} \gamma^{a} \psi_{\mu}=0 \\
\partial_{\mu} \psi_{\nu} & -\partial_{\nu} \psi_{\mu}=0 .
\end{aligned}
$$

The equation for $\psi_{\mu}$ implies that the gravitino field is a pure gauge, being the derivative of a two component Majorana spinor $\psi$ :

$$
\psi_{\mu}=\partial_{\mu} \psi
$$


Analogously, the equation for the dreibein can be simplified, after an integration by parts of the gravitino contribution, as

$$
\partial_{\mu}\left(e_{\nu}^{a}+\frac{1}{16} \bar{\psi} \gamma^{a} \stackrel{\leftrightarrow}{\partial_{\nu}} \psi\right)-\partial_{\nu}\left(e_{\mu}^{a}+\frac{1}{16} \bar{\psi} \gamma^{a} \stackrel{\leftrightarrow}{\partial_{\mu}} \psi\right)=0
$$

Therefore we can define a Minkowskian bosonic coordinate $X^{a}$

$$
e_{\mu}^{a}+\frac{1}{16} \bar{\psi} \gamma^{a} \stackrel{\leftrightarrow}{\partial_{\mu}} \psi=\partial_{\mu} X^{a}
$$

in analogy to gravity.

We have been able to reconstruct, starting from the equations of motion, two primitive functions, one bosonic $X^{a}$ and the other fermionic $\psi$. This corresponds to the well known property that $(2+1)$ supergravity is a pure topological theory, i.e. there are no dynamical degrees of freedom, no free gravitons or gravitinos. The only physical degrees of freedom are those of the external sources ( the simplest ones are a set of $N$ point superparticles ) or topological degrees of freedom. In this paper we will investigate the coupling of $N$-point sources to supergravity, since in this case the supercurvature is concentrated on the point sources. To allow the coupling of a superparticle to supergravity we must require certain polydromic conditions on the $\left(X^{a}, \psi\right)$-mapping, similar to the Deser, Jackiw and 't Hooft $(D J H)$ matching conditions [2]. As a consequence of eqs. (2.9) and (2.11) we will deal with polydromic dreibein and gravitino fields, which are still acceptable since the physical observables are bilinear combination of them ( metrics and currents ), invariant under their monodromies.

\subsection{Mapping $\left(X^{a}, \psi\right)$ as mapping in superspace}

Supergravity has been noted firstly in field theory but then more self-consistent mathematical formalisms have been developed [16]-17], which make use of the concepts of extended coordinates and supermanifolds. Since our method of solution is based on coordinate transformations, we will reformulate the problem of finding a self-consistent solution for the metric and motion of $N$-superparticles in terms of superspace transformations.

At the end of the last paragraph we noted that, in a singular gauge, it was possible to define the coupling with point sources by introducing polydromic conditions on the fields around the particle sites. Here, we want to express these as polydromic conditions on a mapping between supercoordinates, carrying cuts around a set of $N$-point sources:

$$
\begin{aligned}
T & =T(t, z, \bar{z}, \theta, \bar{\theta}) \\
Z & =Z(t, z, \bar{z}, \theta, \bar{\theta}) \\
\Theta & =\Theta(t, z, \bar{z}, \theta, \bar{\theta}) .
\end{aligned}
$$


To generate non-trivial solutions to the $(2+1)$-supergravity field equations, we must carefully choose specific cuts which are compatible with the invariance of the supermetric $d s^{2}$, and therefore related to "isometries" of the flat metric:

$$
d s^{2}=\left(d T+\frac{i}{2}(\Theta d \bar{\Theta}+\bar{\Theta} d \Theta)\right)^{2}-(d Z+\Theta d \Theta)(d \bar{Z}+d \overline{\Theta \Theta})-d \Theta d \bar{\Theta}
$$

Let us note the presence of torsion in superspace that modifies the ordinary differentials to be supersymmetric invariant differentials:

$$
\begin{aligned}
& d T \rightarrow d T+\frac{i}{2}(\Theta d \bar{\Theta}+\bar{\Theta} d \Theta) \\
& d Z \rightarrow d Z+\Theta d \Theta .
\end{aligned}
$$

Moreover we have to know how to relate the data of the superparticle with the parameters of the corresponding monodromy in superspace. In general, the superparticle has to be represented with an extended set of coordinates $\xi^{M}=\left(\xi^{0}, \xi, \bar{\xi}, \xi^{F}, \bar{\xi}^{F}\right)$. The relativistic action of a free superparticle has an internal fermionic symmetry that makes possible, in a particular gauge, to choose the solution of the equations of motion in such a way that the fermionic coordinate is constant:

$$
\xi^{0}=\gamma t \quad \xi(t)=V \xi^{0} \quad \xi^{F}(t)=\epsilon_{0} .
$$

To represent the data of a free superparticle, we must add to the constant velocity $V$ the fermionic constant $\epsilon_{0}$. In the case of gravity we have been able to represent the space-time of $N$ particles as a non trivial mapping between a Minkowskian coordinate system and a physical one $X^{a}=X^{a}\left(x^{b}\right)$. The typical conical singularity of a particle is described by a Lorentz cut in the $X^{a}$ flat coordinate system, i.e. we impose that, when turning around the i-th particle site $\xi_{i}$ in the physical coordinate system $\left(z-\xi_{i}\right) \rightarrow e^{2 \pi i}\left(z-\xi_{i}\right)$

$$
\begin{aligned}
\Delta T & \rightarrow\left(a_{i} \bar{a}_{i}+b_{i} \bar{b}_{i}\right) \Delta T+\bar{a}_{i} b_{i} \Delta Z+a_{i} \bar{b}_{i} \Delta \bar{Z} \\
\Delta Z & \rightarrow 2 \bar{a}_{i} \bar{b}_{i} \Delta T+\bar{a}_{i}^{2} \Delta Z+\bar{b}_{i}^{2} \Delta \bar{Z},
\end{aligned}
$$

where $\Delta T=T-T\left(\xi_{i}\right), \Delta Z=Z-Z\left(\xi_{i}\right)$ are distances between a generic point and the particle site.

We call them $D J H$ matching conditions; these make possible to describe the scattering of point particles in a Minkowskian coordinate system, a property that assures the integrability of the $N$-body problem. In the Minkowskian coordinate system all particles move freely with constant velocities $V_{i},\left(Z\left(\xi_{i}\right)=V_{i} T\left(\xi_{i}\right)+B_{i}\right)$ and the coefficients of the Lorentz cuts are 
related to the particle data as follows [7]

$$
\begin{aligned}
a_{i} & =\cos \frac{m_{i}}{2}+i \gamma_{i} \sin \frac{m_{i}}{2} \\
b_{i} & =-i \gamma_{i} \bar{V}_{i} \sin \frac{m_{i}}{2} \\
V_{i} & \equiv \pm \frac{P_{i}}{E_{i}}, \quad \gamma_{i}=\left(1-\left|V_{i}\right|^{2}\right)^{-\frac{1}{2}}, i=1,2, \ldots, n .
\end{aligned}
$$

Here the $P_{i}^{a}$ are the conserved Minkowskian momenta

$$
P_{i}^{a}=\left(E_{i} / P_{i} / \bar{P}_{i}\right)=m \gamma_{i}\left(1 / V_{i} / \bar{V}_{i}\right)
$$

Is the cut of eq. (2.16) an isometry of the flat metric in superspace? Generally no, unless we modify also the variable $\Theta$ in the following way:

$$
\begin{aligned}
\Theta & \rightarrow \bar{a}_{i} \Theta+i \bar{b}_{i} \bar{\Theta} \\
\bar{\Theta} & \rightarrow a_{i} \bar{\Theta}-i b_{i} \Theta .
\end{aligned}
$$

Then it is not difficult to show that the differentials $d T, d Z$, and those supersymmetric invariant ( $d T+\frac{i}{2}(\Theta d \bar{\Theta}+\bar{\Theta} d \Theta), d Z+\Theta d \Theta$ ) satisfy the same Lorentz polydromy, and therefore in this case the metric $d s^{2}$ remains invariant.

The last property implies that the Lorentz monodromy is compatible with the supersymmetry transformations

$$
\begin{aligned}
d T & \rightarrow d T+\frac{i}{2}\left(d \Theta \bar{\epsilon}_{i}+d \bar{\Theta} \epsilon_{i}\right) \\
d Z & \rightarrow d Z+d \Theta \epsilon_{i} \\
\Theta & \rightarrow \Theta+\epsilon_{i},
\end{aligned}
$$

and the more general monodromy transformations, keeping $d s^{2}$ invariant and defining the coupling of superparticles to supergravity, are:

$$
\begin{aligned}
\Delta T & \rightarrow\left(a_{i} \bar{a}_{i}+b_{i} \bar{b}_{i}\right)\left(\Delta T+\frac{i}{2}\left(\Theta \bar{\epsilon}_{i}+\bar{\Theta} \epsilon_{i}\right)\right)+\bar{a}_{i} b_{i}\left(\Delta Z+\Theta \epsilon_{i}\right)+a_{i} \bar{b}_{i}\left(\Delta \bar{Z}+\bar{\epsilon}_{i} \bar{\Theta}\right) \\
\Delta Z & \rightarrow 2 \bar{a}_{i} \bar{b}_{i}\left(\Delta T+\frac{i}{2}\left(\Theta \bar{\epsilon}_{i}+\bar{\Theta}_{i}\right)\right)+\bar{a}_{i}^{2}\left(\Delta Z+\Theta \epsilon_{i}\right)+\bar{b}_{i}^{2}\left(\Delta \bar{Z}+\bar{\epsilon}_{i} \bar{\Theta}\right) \\
\Theta & \rightarrow \bar{a}_{i}\left(\Theta+\epsilon_{i}\right)+i \bar{b}_{i}\left(\bar{\Theta}+\bar{\epsilon}_{i}\right),
\end{aligned}
$$

where as before $\Delta T=T-T\left(\xi_{i}\right), \Delta Z=Z-Z\left(\xi_{i}\right)$ and $Z\left(\xi_{i}\right)-V_{i} T\left(\xi_{i}\right)=B_{i}$. Let us note the following remarkable property of these monodromies, i.e the fact that, by introducing 
supersymmetric invariant distances

$$
\begin{aligned}
\Delta_{S} T & =T-T\left(\xi_{i}\right)-\frac{i}{2}\left(\Theta \bar{\Theta}\left(\xi_{i}\right)+\bar{\Theta} \Theta\left(\xi_{i}\right)\right) \\
\Delta_{S} Z & =Z-Z\left(\xi_{i}\right)-\Theta \Theta\left(\xi_{i}\right) \\
\Delta_{S} \Theta & =\Theta-\Theta\left(\xi_{i}\right)
\end{aligned}
$$

these can be recast in a more elegant form

$$
\begin{aligned}
\Delta_{S} T & \rightarrow\left(a_{i} \bar{a}_{i}+b_{i} \bar{b}_{i}\right) \Delta_{S} T+\bar{a}_{i} b_{i} \Delta_{S} Z+a_{i} \bar{b}_{i} \Delta_{S} \bar{Z} \\
\Delta_{S} Z & \rightarrow 2 \bar{a}_{i} \bar{b}_{i} \Delta_{S} T+\bar{a}_{i}^{2} \Delta_{S} Z+\bar{b}_{i}^{2} \Delta_{S} \bar{Z} \\
\Delta_{S} \Theta & \rightarrow \bar{a}_{i} \Delta_{S} \Theta+i \bar{b}_{i} \Delta_{S} \bar{\Theta}
\end{aligned}
$$

where $\Theta\left(\xi_{i}\right)$ are defined as fixed points of the $\Theta$ monodromy.

To be more precise, the correspondence is not completely exact. In fact, there is a missing piece $O(\epsilon \bar{\epsilon})$, between eq. (2.21) and eq. (2.23). If we believe in the last equation, which is manifestly supersymmetric f, we have to correct eq. (2.21) as:

$$
\begin{aligned}
\Delta T & \rightarrow\left(a_{i} \bar{a}_{i}+b_{i} \bar{b}_{i}\right)\left(\Delta T+\frac{i}{2}\left(\Theta \bar{\epsilon}_{i}+\bar{\Theta}_{i}\right)\right)+\bar{a}_{i} b_{i}\left(\Delta Z+\Theta \epsilon_{i}\right)+a_{i} \bar{b}_{i}\left(\Delta \bar{Z}+\bar{\epsilon}_{i} \bar{\Theta}\right) \\
& +\frac{i}{2}(a-\bar{a}) \Theta\left(\xi_{i}\right) \bar{\Theta}\left(\xi_{i}\right) \\
\Delta Z & \rightarrow 2 \bar{a}_{i} \bar{b}_{i}\left(\Delta T+\frac{i}{2}\left(\Theta \bar{\epsilon}_{i}+\bar{\Theta}_{i}\right)\right)+\bar{a}_{i}^{2}\left(\Delta Z+\Theta \epsilon_{i}\right)+\bar{b}_{i}^{2}\left(\Delta \bar{Z}+\bar{\epsilon}_{i} \bar{\Theta}\right)+i \bar{b} \Theta\left(\xi_{i}\right) \bar{\Theta}\left(\xi_{i}\right) \\
\Theta & \rightarrow \bar{a}_{i}\left(\Theta+\epsilon_{i}\right)+i \bar{b}_{i}\left(\bar{\Theta}+\bar{\epsilon}_{i}\right) .
\end{aligned}
$$

We have discovered a translation monodromy, not to be confused with the typical translation monodromy of the spinning sources [11].

We can also write down a more general case, in which a real spinning source $S_{i}$ is present:

$$
\begin{aligned}
\Delta_{S} X^{a} & \rightarrow L_{i b}^{a} \Delta_{S} X^{b}+\frac{S_{i}}{m_{i}} P_{i}^{a} \\
\Delta_{S} \Theta & \rightarrow \bar{a}_{i} \Delta_{S} \Theta+i \bar{b}_{i} \Delta_{S} \bar{\Theta}
\end{aligned}
$$

We will see that this extra term is unavoidable in the explicit solution and it will give rise to a logarithmic behaviour of the $X^{M}$ supercoordinates around the superparticle sites, breaking the manifest supersymmetry of eq (2.23).

Finally, let us note that, starting from the knowledge of the coordinate transformation in superspace, we can deduce the dreibein, which is polydrome only with respect to the Lorentz part of the monodromy

${ }^{*}$ We also must require that when $X^{M}=X^{M}\left(\xi_{i}\right)$ the monodromy reduces to $0 \rightarrow 0$. 


$$
\begin{aligned}
e_{\mu}^{a} & =\left(\partial_{\mu} T+\frac{i}{2}\left(\Theta \partial_{\mu} \bar{\Theta}+\bar{\Theta} \partial_{\mu} \Theta\right), \partial_{\mu} Z+\Theta \partial_{\mu} \Theta, \partial_{\mu} \bar{Z}+\partial_{\mu} \overline{\Theta \Theta}\right) \\
\psi_{\mu} & =\partial_{\mu} \Theta
\end{aligned}
$$

This parameterization of the solution makes explicit that the source of torsion in the dreibein equation is solved by the presence of bilinear terms in $\Theta$. From now on we will concentrate only on computing the supercoordinate transformation, which is enough to solve the superparticle motion.

\subsection{Solution for the cuts}

In order to define the supermapping (2.12), we have to impose some gauge condition. In gravity we have learned that it exists a gauge condition for the metric in which all the metric components propagate instantaneously [6] and that space-time can be foliated in terms of space-like hypersurfaces as in the ADM formalism [19]. It can also be defined in a first order formalism as a $X^{a}=X^{a}\left(x^{b}\right)$ mapping that can be decomposed as a sum of analytic and antianalytic functions [7]. We are going to extend the second definition of instantaneous gauge to the case of supergravity, since searching a generalization in the first order formalism, based on the dreibein, is simpler than looking for a gauge condition for the metric in this theory. In fact we can provide a realization of the cuts for the coordinates $T, Z, \Theta$ starting from analytic functions

$$
\begin{aligned}
T & =T(t)+A_{0}(z)+\overline{A_{0}}(\bar{z})+O(\theta, \bar{\theta}) \\
Z & =A_{1}(z)+\overline{A_{2}}(\bar{z})+O(\theta, \bar{\theta}) \\
\Theta & =\psi(z)+\bar{\chi}(\bar{z})+O(\theta, \bar{\theta})
\end{aligned}
$$

Solving the DJH matching conditions around the superparticles implies the following conditions on the cuts of the analytic functions

$$
\begin{aligned}
A_{0}^{\prime}(z) & \rightarrow(a \bar{a}+b \bar{b})\left(A_{0}^{\prime}(z)+\frac{i}{2}\left(\psi^{\prime}(z) \bar{\epsilon}+\chi^{\prime}(z) \epsilon\right)\right)+\bar{a} b\left(A^{\prime}{ }_{1}(z)+\psi^{\prime}(z) \epsilon\right)+a \bar{b}\left(A_{2}^{\prime}(z)+\bar{\epsilon} \chi^{\prime}(z)\right) \\
A^{\prime}{ }_{1}(z) & \rightarrow 2 \bar{a} \bar{b}\left(A^{\prime}{ }_{0}(z)+\frac{i}{2}\left(\psi^{\prime}(z) \bar{\epsilon}+\chi^{\prime}(z) \epsilon\right)\right)+\bar{a}^{2}\left(A_{1}^{\prime}(z)+\psi^{\prime}(z) \epsilon\right)+\bar{b}^{2}\left(A^{\prime}{ }_{2}(z)+\bar{\epsilon} \chi^{\prime}(z)\right) \\
A^{\prime}{ }_{2}(z) & \rightarrow 2 a b\left(A^{\prime}{ }_{0}(z)+\frac{i}{2}\left(\psi^{\prime}(z) \bar{\epsilon}+\chi^{\prime}(z) \epsilon\right)\right)+b^{2}\left(A_{1}^{\prime}(z)+\psi^{\prime}(z) \epsilon\right)+a^{2}\left(A^{\prime}{ }_{2}(z)+\bar{\epsilon} \chi^{\prime}(z)\right) \\
\psi(z) & \rightarrow \bar{a}\left(\psi(z)+\frac{\epsilon}{2}\right)+i \bar{b}\left(\chi+\frac{\bar{\epsilon}}{2}\right)
\end{aligned}
$$




$$
\chi(z) \rightarrow a\left(\chi(z)+\frac{\bar{\epsilon}}{2}\right)-i b\left(\psi+\frac{\epsilon}{2}\right) .
$$

In the last two equations we made the hypothesis that the supersymmetric translation of $\Theta$ is divided in equal parts between $\psi$ and $\chi$. The monodromy problem (2.28) seems too difficult to solve, as it is formulated. Our strategy will be to define supersymmetric invariant analytic functions, which turn out to be useful for reducing this system to a more manageable one.

In fact, being $\left(\psi^{\prime}, \chi^{\prime}\right)$ invariants under supersymmetric cuts, we can integrate those defining some new combinations which are again covariant under Lorentz transformations

$$
\begin{aligned}
& A_{0}^{\prime}+i\left(\chi \psi^{\prime}+\psi \chi^{\prime}\right) \rightarrow(a \bar{a}+b \bar{b})\left(A_{0}^{\prime}+i\left(\chi \psi^{\prime}+\psi \chi^{\prime}\right)\right)+\bar{a} b\left(A_{1}^{\prime}+2 \psi \psi^{\prime}\right)+a \bar{b}\left(A_{2}^{\prime}+2 \chi^{\prime} \chi\right) \\
& A_{1}^{\prime}+2 \psi \psi^{\prime} \rightarrow 2 \bar{a} \bar{b}\left(A_{0}^{\prime}+i\left(\chi \psi^{\prime}+\psi \chi^{\prime}\right)\right)+\bar{a}^{2}\left(A_{1}^{\prime}+2 \psi \psi^{\prime}\right)+\bar{b}^{2}\left(A_{2}^{\prime}+2 \chi^{\prime} \chi\right) \\
& A_{2}^{\prime}+2 \chi^{\prime} \chi \rightarrow 2 a b\left(A_{0}^{\prime}+i\left(\chi \psi^{\prime}+\psi \chi^{\prime}\right)\right)+b^{2}\left(A_{1}^{\prime}+2 \psi \psi^{\prime}\right)+a^{2}\left(A_{2}^{\prime}+2 \chi^{\prime} \chi\right) .
\end{aligned}
$$

Thus, we can identify these analytic functions with the ones introduced in $(2+1)$ gravity

$$
\begin{aligned}
& A^{\prime}{ }_{0}(z)+i\left(\chi(z) \psi^{\prime}(z)+\psi(z) \chi^{\prime}(z)\right)=\frac{N(z)}{f^{\prime}(z)} f(z) \\
& A^{\prime}{ }_{1}(z)+2 \psi(z) \psi^{\prime}(z)=\frac{N(z)}{f^{\prime}(z)} \\
& A^{\prime}{ }_{2}(z)+2 \chi^{\prime}(z) \chi(z)=\frac{N(z)}{f^{\prime}(z)} f^{2}(z) .
\end{aligned}
$$

The function $N(z, t)$ is a meromorphic function with poles at the singularities and for the two-body problem was found to be [7]:

$$
N(z, t)=C \xi^{1-\frac{\mathcal{M}}{2 \pi}} \frac{1}{\left(z-\xi_{1}\right)\left(\xi_{2}-z\right)} \quad \xi=\xi_{2}-\xi_{1},
$$

and the function $f(z, t)$ can be exactly given for the two-body problem (see Appendix), and perturbatively for the $N$-body problem. In the two body case the function has branch points at $\zeta=0$ and $\zeta=1$ ( and $\zeta=\infty$ ), around which it has to transform projectively as

$$
f(z, t) \rightarrow \frac{a_{i} f(z, t)+b_{i}}{\bar{b}_{i} f(z, t)+\bar{a}_{i}}
$$

The $f$ function is obtained as a ratio of two independent solutions $y_{1}$ and $y_{2}$ of the 
Fuchsian equation in the adimensional variable $\zeta=\left(z-\xi_{1}\right) /\left(\xi_{2}-\xi_{1}\right)$

$$
y^{\prime \prime}+\frac{1}{4}\left(\frac{1-\mu_{1}^{2}}{\zeta^{2}}+\frac{1-\mu_{2}^{2}}{(1-\zeta)^{2}}+\frac{1-\mu_{1}^{2}-\mu_{2}^{2}+\mu_{\infty}^{2}}{\zeta(1-\zeta)}\right) y=0 .
$$

The indices $\mu_{1}, \mu_{2}$ and $\mu_{\infty}$, appearing in eq. (2.33), are related to the masses $m_{1}, m_{2}$ and the physical invariant mass $\mathcal{M}$ as follows

$$
\begin{aligned}
\mu_{1} & =\frac{m_{1}}{2 \pi}, \quad \mu_{2}=\frac{m_{2}}{2 \pi} \\
\mu_{\infty} & =\left(\frac{\mathcal{M}}{2 \pi}-1\right),
\end{aligned}
$$

where $\mathcal{M}$, corresponding to the topological invariant $\operatorname{Tr}\left(L_{1} L_{2}\right)$, is given by the Carlip's formula 20

$$
\cos \frac{\mathcal{M}}{2}=\cos \frac{m_{1}}{2} \cos \frac{m_{2}}{2}-\frac{P_{1} \cdot P_{2}}{m_{1} m_{2}} \sin \frac{m_{1}}{2} \sin \frac{m_{2}}{2} .
$$

In the case of $N$-body the function $f(z, t)$ is determined again by the same procedure, however the Schwarzian in this case contains not only poles at the $(N+1)$ physical singularities but also poles at $N-2$ apparent singularities, whose motion, as a function of the physical poles, is constrained by the requirement of isomonodromy of the monodromy matrices, as discussed in [7]- 8]-21].

The non-triviality of supergravity is hidden in finding a solution for the gravitino fields $\psi$ and $\chi$, which is simpler than the starting problem in eq. (2.28), since we have to look for only a spin $\frac{1}{2}$ representation of the monodromies. It was exactly this reduction that has permitted us to write the non perturbative solution for the two body problem. In order to determine $\psi$ and $\chi$ we have to solve the monodromy conditions

$$
\begin{aligned}
& \psi \rightarrow \bar{a}\left(\psi+\frac{\epsilon}{2}\right)+i \bar{b}\left(\chi+\frac{\bar{\epsilon}}{2}\right) \\
& \chi \rightarrow a\left(\chi+\frac{\bar{\epsilon}}{2}\right)-i b\left(\psi+\frac{\epsilon}{2}\right) .
\end{aligned}
$$

The terms of order $\theta$ in the $X$-supermapping (2.27) can be built from combinations of the functions of order zero in $\theta$ that respect the Lorentz and supersymmetric cuts, from which we obtain the complete solution in superspace

$$
\begin{aligned}
T & =T(t)+A_{0}(z)+\bar{A}_{0}(\bar{z})+\theta\left[i k_{0} \chi \sqrt{A_{1}^{\prime}+2 \psi \psi^{\prime}}+k_{0} \psi \sqrt{A_{2}^{\prime}+2 \chi^{\prime} \chi}\right] \\
& +\left[-i \bar{k}_{0} \bar{\chi} \sqrt{{\overline{A^{\prime}}}_{1}+2 \bar{\psi}^{\prime} \bar{\psi}}+\bar{k}_{0} \bar{\psi} \sqrt{\overline{A^{\prime}}{ }_{2}+2 \overline{\chi \chi}^{\prime}}\right] \bar{\theta}
\end{aligned}
$$




$$
\begin{aligned}
& -i \theta \bar{\theta}\left[h_{0}\left(\psi \chi^{\prime}+\chi \psi^{\prime}\right)+\bar{h}_{0}\left(\overline{\psi \chi^{\prime}}+\bar{\chi} \overline{\psi^{\prime}}\right)\right] \\
Z & =A_{1}(z)+\overline{A_{2}}(\bar{z})+2 \theta k_{0} \psi \sqrt{A^{\prime}{ }_{1}+2 \psi \psi^{\prime}}-2 i \bar{k}_{0} \bar{\chi} \sqrt{{\overline{A^{\prime}}}_{2}+2 \overline{\chi \chi}} \bar{\gamma}^{\prime} \\
- & 2 \theta \bar{\theta}\left[h_{0} \psi \psi^{\prime}+\bar{h}_{0} \bar{\chi} \overline{\chi^{\prime}}\right] \\
\Theta & =\psi(z)+\bar{\chi}(\bar{z})+\theta k_{0} \sqrt{A^{\prime}{ }_{1}+2 \psi \psi^{\prime}}+i \overline{\theta k}_{0} \sqrt{{\overline{A^{\prime}}}_{2}+2 \overline{\chi \chi}^{\prime}} \\
& +\theta \bar{\theta}\left(h_{0} \psi^{\prime}+\bar{h}_{0} \overline{\chi^{\prime}}\right),
\end{aligned}
$$

where $k_{0}$ and $h_{0}$ are arbitrary constant which are left undetermined by the monodromies. In the following we will set, for simplicity, $k_{0}=1$ which is equivalent to a rescaling of $\theta \rightarrow \theta / k_{0}$. To derive eq. (2.37) we have been inspired by the form of superanalytic functions [?.

Naively, this definition of the $X$-supermapping can be shown to satisfy the rule (2.23). In fact, the combinations $\Delta_{S} X^{a}$ can be written as follows:

$$
\begin{aligned}
\Delta_{S} T & =\int_{\xi_{i}}^{z}\left(\frac{N}{f^{\prime}} f-i\left(\Delta_{S} \psi \chi^{\prime}+\Delta_{S} \chi \psi^{\prime}\right)\right) d z+\theta\left[i \Delta_{S} \chi \sqrt{\frac{N}{f^{\prime}}}+\Delta_{S} \psi \sqrt{\frac{N}{f^{\prime}} f}\right]+ \\
& -i \quad \theta \bar{\theta} h_{0}\left(\Delta_{S} \psi \chi^{\prime}+\Delta_{S} \chi \psi^{\prime}\right)+(h . c .) \\
\Delta_{S} Z & =\int_{\xi_{i}}^{z}\left(\frac{N}{f^{\prime}}-2 \Delta_{S} \psi \psi^{\prime}\right) d z+\int_{\bar{\xi}_{i}}^{\bar{z}}\left(\frac{\bar{N}}{\overline{f^{\prime}}} \bar{f}-2 \Delta_{S} \overline{\chi^{\prime}}(\bar{z})\right) d \bar{z}+2 \theta \Delta_{S} \psi \sqrt{\frac{N}{f^{\prime}}}- \\
& -2 i \Delta_{S} \bar{\chi} \sqrt{\frac{\bar{N}}{\overline{f^{\prime}}} \overline{f \theta}}-2 \theta \bar{\theta}\left[h_{0} \Delta_{S} \psi \psi^{\prime}+\bar{h}_{0} \Delta_{S} \bar{\chi} \overline{\chi^{\prime}}\right] \\
\Delta_{S} \Theta & =\Delta_{S} \psi(z)+\Delta_{S} \bar{\chi}(\bar{z})+\theta \sqrt{\frac{N}{f^{\prime}}}+i \bar{\theta} \sqrt{\frac{\bar{N}}{\overline{f^{\prime}}} \bar{f}}+\theta \bar{\theta}\left(h_{0} \psi^{\prime}+\bar{h}_{0} \overline{\chi^{\prime}}\right) .
\end{aligned}
$$

In this formula we have introduced the notations $\Delta_{S} \psi=\psi(z)-\psi\left(\xi_{i}\right)$ and $\Delta_{S} \chi=\chi(z)-\chi\left(\xi_{i}\right)$, where $\psi\left(\xi_{i}\right)$ and $\chi\left(\xi_{i}\right)$ are defined as fixed points of the $\psi$ and $\chi$ monodromies. Since $\Delta_{S} \psi$ and $\Delta_{S} \chi$ transform covariantly under Lorentz transformations without translation terms, the whole mapping formally obeys eq. (2.23), unless the integration of $1 / z$ terms produces logarithms spoiling supersymmetry covariance. It will turn out, by analyzing in detail the one-body problem, that the explicit solution breaks this covariance.

\section{Two-superbody problem}

In this section we will take advantage of this explicit parameterization in order to have detailed informations about superparticle dynamics. Let us start with the one-body static

\footnotetext{
${ }^{\dagger}$ We have avoided to consider terms which do not respect the condition that, when the source is decoupled $(\epsilon=m=0)$, the supermapping reduces to the identity. This requirement completes our gauge choice.
} 
problem and notice that, at the singularity, the value of $\Theta$ is fixed by the monodromy conditions

$$
\Theta(0)=\frac{\epsilon}{a-1}
$$

Since from gravity we know that $f=0$, and $N / f^{\prime}=z^{-\mu}$, it remains to look for a solution to the system (2.36), of the type

$$
\psi^{\prime}=\delta_{1} z^{-\frac{\mu}{2}-1} \quad \chi^{\prime}=\delta_{2} z^{\frac{\mu}{2}-1} .
$$

Requiring that $\psi \rightarrow \bar{a}\left(\psi+\frac{\epsilon}{2}\right)$ and $\chi \rightarrow a\left(\chi+\frac{\bar{\epsilon}}{2}\right)$ fixes only the boundary values $\psi(0)=$ $\bar{\chi}(0)=\Theta(0) / 2$, and leaves $\delta_{1}$ and $\delta_{2}$ undetermined:

$$
\begin{aligned}
& \psi(z)=\psi(0)-\frac{2}{\mu} \delta_{1} z^{-\frac{\mu}{2}} \\
& \chi(z)=\chi(0)+\frac{2}{\mu} \delta_{2} z^{\frac{\mu}{2}} .
\end{aligned}
$$

Substituting these expressions into eq. (2.37), we can compute the total supercoordinate transformation as:

$$
\begin{aligned}
T(z, \bar{z}, t) & =T_{0}(t)-i \int_{0}^{z} d z\left(\psi \chi^{\prime}+\chi \psi^{\prime}\right)+\theta i \chi \sqrt{\frac{N}{f^{\prime}}}-i \theta \bar{\theta} h_{0}\left(\psi \chi^{\prime}+\chi \psi^{\prime}\right)+(h . c .)= \\
& =T_{0}(t)+\frac{2 i}{\mu} \chi(0) \delta_{1} z^{-\frac{\mu}{2}}-\frac{2 i}{\mu} \psi(0) \delta_{2} z^{\frac{\mu}{2}}+\frac{4 i}{\mu} \delta_{1} \delta_{2} \log z+ \\
& -i \chi(0) \theta z^{-\frac{\mu}{2}}+\frac{2 i}{\mu} \theta \delta_{2}-i \theta \bar{\theta} h_{0}\left(\psi(0) \delta_{2} z^{\frac{\mu}{2}-1}+\chi(0) \delta_{1} z^{-\frac{\mu}{2}-1}\right)+(h . c .) \\
Z(z) & =\frac{z^{1-\mu}}{1-\mu}+\frac{4}{\mu} \psi(0) \delta_{1} z^{-\frac{\mu}{2}}-\frac{4}{\mu} \bar{\chi}(0) \bar{\delta}_{2} \bar{z}^{\frac{\mu}{2}}+ \\
& +2 \theta \psi(0) z^{-\frac{\mu}{2}}-\frac{4}{\mu} \theta \delta_{1} z^{-\mu}-2 \theta \bar{\theta}\left(h_{0} \psi(0) \delta_{1} z^{-\frac{\mu}{2}-1}+\bar{h}_{0} \bar{\chi}(0) \bar{\delta}_{2} \bar{z}^{\frac{\mu}{2}-1}\right) \\
\Theta(z) & =\Theta(0)-\frac{2}{\mu} \delta_{1} z^{-\frac{\mu}{2}}+\frac{2}{\mu} \bar{\delta}_{2} \bar{z}^{\frac{\mu}{2}}+\theta z^{-\frac{\mu}{2}} \\
& +\theta \bar{\theta}\left(h_{0} \delta_{1} z^{-\frac{\mu}{2}-1}+\bar{h}_{0} \bar{\delta}_{2} \bar{z}^{\frac{\mu}{2}-1}\right) .
\end{aligned}
$$

Around zero, the logarithmic contribution gives rise to an additional translation monodromy for $\mathrm{T}$, which has no relation with the translation of (eq. (2.24) ) since it comes from the integration of an unwanted $1 / z$ term,

$$
\Delta_{S} T \rightarrow \Delta_{S} T-\frac{16}{\mu} R e\left(\delta_{1} \delta_{2}\right)
$$

and instead reveals the presence of a spin source $S \sim R e\left(\delta_{1} \delta_{2}\right)$, as in eq. (2.25). Since this definition obeys the condition $S^{2}=0$, it is possible to avoid the $C T C$ problem, which was 
encountered in the solution of spinning particles, with the spin variable treated as a pure classical variable. We interpret it not as an external spinning source but as a spontaneous violation of supersymmetry in presence of a conical singularity.

Since at the singularity the value of $\Theta(0)$ is trivially reproduced, apparently there is no condition to constraint the fermionic trajectory. Here there is a difference from the bosonic case, where requiring that the $X^{a}$ mapping has a specific value at the particle site $X^{a}\left(\xi_{i}\right)$ was enough to determine the general first integral of the geodesic equations. However there is a singularity of the mapping at the particle site, as in the case of spinning particles coupled to gravity, $\psi(z) \sim z^{-\frac{\mu}{2}}$. Moreover, in this case the superspace geometry can help to give an interesting interpretation to it. In fact, to understand better the role of this divergence we must add the contribution in $\theta$ which dresses the divergent part in $\psi(z)$

$$
\Theta=\left(\theta-\frac{2}{\mu} \delta_{1}\right) z^{-\frac{\mu}{2}}+\theta \bar{\theta}\left(h_{0} \delta_{1} z^{-\frac{\mu}{2}-1}+\bar{h}_{0} \bar{\delta}_{2} \bar{z}^{\frac{\mu}{2}-1}\right) \ldots
$$

To have a well defined theory at the classical level, we require that the singular behaviour disappears, due to the contribution of $\theta(0)$ :

$$
\theta(0)=\xi^{F}=\frac{2}{\mu} \delta_{1}
$$

This is the obvious solution to the spinor part of the supergeodesics equations [16] for a static superparticle. The spinor coordinate $\xi^{F}$ is ill-defined when the mass $m \rightarrow 0$ but this is correct because a massless particle at rest, carrying an additional intrinsic degree of freedom ( the constant spinor $\epsilon$ ), is not a self-consistent concept.

The contribution at the singularity of the type $z^{-\frac{\mu}{2}}$ in the bosonic coordinates $T, Z, \bar{Z}$ is again cancelled due to eq. (3.7), whatever the value of $\delta_{1}$ is. In fact for the $\mathrm{T}$ coordinate we obtain the singular contribution:

$$
\left.T\right|_{\operatorname{sing}} \sim-i \chi(0)\left(\theta-\frac{2}{\mu} \delta_{1}\right) z^{-\frac{\mu}{2}},
$$

and similarly for the $Z$ coordinate

$$
\left.Z\right|_{\text {sing }} \sim 2\left(\theta-\frac{2}{\mu} \delta_{1}\right) \psi(0) z^{-\frac{\mu}{2}} .
$$

The $\theta \bar{\theta}$ terms induce other divergences $z^{ \pm \frac{\mu}{2}-1}$, which cannot be mixed with $z^{-\frac{\mu}{2}}$. To get a cancellation of them we should require that $\delta_{2}$ is a linear combination of $\delta_{1}$ and $\bar{\delta}_{1}$. It is of course possible to restrict the arbitrary parameters $\delta_{1}$ and $\delta_{2}$ to do so, but, for more than one particle, this requirement would lead to an involved solution, carrying many parameters. 
To keep the discussion as more concise as possible, we will set in the following the arbitrary parameter $h_{0}=0$, since then a minimal solution ( but not trivial ) is shown to be consistent at all orders.

In conclusion, while in the bosonic case the finite values $X^{a}\left(x_{i}\right)$ are responsible for the $\xi(t)$ bosonic trajectory, in the fermionic case we find that the divergent behaviour of the $X^{M}$ mapping around the particle site defines the $\xi^{F}(t)$ fermionic trajectory and the finite values $\Theta\left(\xi_{i}\right)$ are trivially reobtained by the explicit solution.

The two-body static case is similarly obtained by the monodromies :

$$
\begin{array}{ll}
\psi \stackrel{1}{\rightarrow} \bar{a}_{1}\left(\psi+\frac{\epsilon_{1}}{2}\right) & \chi \stackrel{1}{\rightarrow} a_{1}\left(\chi+\frac{\overline{\epsilon_{1}}}{2}\right) \\
\psi \stackrel{1}{\rightarrow} \bar{a}_{2}\left(\psi+\frac{\epsilon_{2}}{2}\right) & \chi \stackrel{2}{\rightarrow} a_{2}\left(\chi+\frac{\overline{\epsilon_{2}}}{2}\right) .
\end{array}
$$

Let us suppose to have two superparticles at rest $\left(z=\xi_{i}\right)$, which can be mapped by introducing the adimensional variable $\zeta=\left(z-\xi_{1}\right) /\left(\xi_{2}-\xi_{1}\right)$, into the points $\zeta=0$ and $\zeta=1$. At the singularity, the value of $\Theta$ is fixed by the monodromy

$$
\Theta(0)=2 \psi(0)=2 \bar{\chi}(0)=\frac{\epsilon_{1}}{a_{1}-1} \quad \Theta(1)=2 \psi(1)=2 \bar{\chi}(1)=\frac{\epsilon_{2}}{a_{2}-1} .
$$

Let us look for a solution of the type:

$$
\begin{aligned}
& \psi^{\prime}=\left[\frac{\delta_{1}}{\zeta}+\frac{\sigma_{1}}{1-\zeta}\right] \zeta^{-\frac{\mu_{1}}{2}}(1-\zeta)^{-\frac{\mu_{2}}{2}} \\
& \chi^{\prime}=\left[\frac{\delta_{2}}{\zeta}+\frac{\sigma_{2}}{1-\zeta}\right] \zeta^{\frac{\mu_{1}}{2}}(1-\zeta)^{\frac{\mu_{2}}{2}}
\end{aligned}
$$

The condition which determines the unknowns $\delta_{i}, \sigma_{i}(i=1,2)$ are the fermionic distances, invariant under constant fermionic translations of the $\Theta$-variable,

$$
\begin{aligned}
& \psi(1)-\psi(0)=\int_{0}^{1} d \zeta \psi^{\prime}=-2 \frac{\Gamma\left(1-\frac{\mu_{1}}{2}\right) \Gamma\left(1-\frac{\mu_{2}}{2}\right)}{\Gamma\left(1-\frac{\mu_{1}}{2}-\frac{\mu_{2}}{2}\right)}\left[\frac{\delta_{1}}{\mu_{1}}+\frac{\sigma_{1}}{\mu_{2}}\right] \\
& \chi(1)-\chi(0)=\int_{0}^{1} d \zeta \chi^{\prime}=2 \frac{\Gamma\left(1+\frac{\mu_{1}}{2}\right) \Gamma\left(1+\frac{\mu_{2}}{2}\right)}{\Gamma\left(1+\frac{\mu_{1}}{2}+\frac{\mu_{2}}{2}\right)}\left[\frac{\delta_{2}}{\mu_{1}}+\frac{\sigma_{2}}{\mu_{2}}\right] .
\end{aligned}
$$

The monodromy conditions for $\psi$ and $\chi$ leave always two free parameters, in the general $N$-body case too. We can of course determine the solution by adding some "physical requirements", but we prefer to take into account only the necessary ones, such as the monodromies. In any case this formalism can be generalized to support more constraints on the anticommuting variables, by increasing the number of parameters. 
As in the one-body problem, unavoidable logarithmic terms appear as follows

$$
\begin{aligned}
T & \stackrel{\zeta \sim 0}{\sim} \frac{4 i}{\mu_{1}} \delta_{1} \delta_{2} \log \zeta+(\text { h.c. }) \quad \Rightarrow \Delta_{S} T \stackrel{1}{\rightarrow} \Delta_{S} T-\frac{16}{\mu_{1}} \operatorname{Re}\left(\delta_{1} \delta_{2}\right) \\
& \stackrel{\zeta \sim 1}{\sim} \frac{4 i}{\mu_{2}} \sigma_{1} \sigma_{2} \log (\zeta-1)+(\text { h.c. }) \quad \Rightarrow \Delta_{S} T \stackrel{2}{\rightarrow} \Delta_{S} T-\frac{16}{\mu_{2}} \operatorname{Re}\left(\sigma_{1} \sigma_{2}\right),
\end{aligned}
$$

revealing the presence of a spin $S_{1} \sim \operatorname{Re}\left(\delta_{1} \delta_{2}\right)$ at $\zeta=0$ and $S_{2} \sim \operatorname{Re}\left(\sigma_{1} \sigma_{2}\right)$ at $\zeta=1$.

Moreover there is a singular behaviour of the field $\psi$, which is unphysical at the classical level. As in the one body case, we obtain a cancellation of the divergence at the particle site by adding the contribution in $\theta$ :

$$
\Theta=\psi(z)+\bar{\chi}(\bar{z})+\theta \sqrt{\frac{N}{f^{\prime}}}+O(\theta \bar{\theta})
$$

In the static case $f=0, \sqrt{\frac{N}{f^{\prime}}}=\left(z-\xi_{1}\right)^{-\frac{\mu_{1}}{2}}\left(\xi_{2}-z\right)^{-\frac{\mu_{2}}{2}}$, therefore the $\theta$ term dresses the divergent part in $\psi_{0}(z)$

$$
\left.\Theta\right|_{\zeta \simeq 0}=\xi^{-\frac{\mu_{2}}{2}}\left(\theta+\frac{2}{\mu_{1}} \delta_{1} \xi^{\frac{\mu_{1}+\mu_{2}}{2}}\right)\left(z-\xi_{1}\right)^{-\frac{\mu_{1}}{2}}
$$

and defines the fermionic coordinates:

$$
\theta(0)=\xi_{1}^{F}=-\frac{2}{\mu_{1}} \delta_{1} \xi^{\frac{\mu_{1}+\mu_{2}}{2}} \quad \theta(1)=\xi_{2}^{F}=\frac{2}{\mu_{2}} \sigma_{1} \xi^{\frac{\mu_{1}+\mu_{2}}{2}}
$$

It is interesting to observe that the difference $\xi_{2}^{F}-\xi_{1}^{F}$ is physically meaningful

$$
\theta(1)-\theta(0)=-\frac{\Gamma\left(1-\frac{\mu_{1}}{2}-\frac{\mu_{2}}{2}\right)}{\Gamma\left(1-\frac{\mu_{1}}{2}\right) \Gamma\left(1-\frac{\mu_{2}}{2}\right)} \xi^{\frac{\mu_{1}+\mu_{2}}{2}}(\psi(1)-\psi(0)),
$$

being dependent only on the constant of motions. Therefore the aforementioned arbitrariness could be related to the choice of a fermionic center of mass, while the relative coordinate is unambiguous.

Let us check how the divergent terms $\left(z-\xi_{i}\right)^{-\frac{\mu_{i}}{2}}$ are cancelled by the $\theta$ contributions in the bosonic $X^{a}$ mappings. For example the divergence of the type $\left(z-\xi_{1}\right)^{-\frac{\mu_{1}}{2}}$ in the $T$ and $Z$-variables can be written as follows

$$
T \stackrel{1}{\sim}-i \chi(0)\left(\psi+\theta \sqrt{\frac{N}{f^{\prime}}}\right) \quad Z \stackrel{1}{\sim}-2 \psi(0)\left(\psi+\theta \sqrt{\frac{N}{f^{\prime}}}\right),
$$


since $\psi$ and $\chi$ can be considered constant. Again the cancellation of the fermionic residue at the singularity leads to equation (3.17).

The bosonic part of the geodetic equations is equivalent to impose $Z\left(\xi_{2}\right)-Z\left(\xi_{1}\right)=B_{2}-B_{1}$ and is of course trivial since only determines the distance $\xi$ in terms of the bosonic constant of motion $B_{2}-B_{1}$.

\subsection{Non-perturbative solution}

There are many non perturbative informations that can be extracted without doing any calculations, in practice. By using eq. (2.21) the value of $\Theta$ at the particle site is determined as a fixed point of the $\Theta$ monodromy as

$$
\Theta_{i}=2 \psi\left(\xi_{i}\right)=2 \bar{\chi}\left(\xi_{i}\right)=\frac{\left(\bar{a}_{i}-1\right) \epsilon_{i}+i \bar{b}_{i} \bar{\epsilon}_{i}}{2-a_{i}-\bar{a}_{i}} .
$$

Moreover we note that the $\theta$-terms cannot give any finite contribution at the particle sites in the $\Theta$-mapping (2.37). Therefore, the conclusion that the fermionic geodetic cannot be obtained by means of finite terms is simply a corollary of the monodromy properties.

Without solving exactly the fermionic fields, it is possible to discuss in full detail the fermionic geodetic equation, since the monodromies tell us enough information to constraint the behaviour of these fields around the superparticles.

In particular, there are two leading behaviours $\zeta^{ \pm \frac{\mu_{1}}{2}}$ around the superparticles and their coefficients are constrained in the following way

$$
\begin{aligned}
\psi-\psi(0) & \simeq \Delta_{1} \zeta^{-\frac{\mu_{1}}{2}}+i \bar{f}(0) \Delta_{2} \zeta^{\frac{\mu_{1}}{2}}+O\left(\zeta^{1-\frac{\mu_{1}}{2}}\right)+O\left(\zeta^{1+\frac{\mu_{1}}{2}}\right) \\
\chi-\chi(0) & \simeq-i f(0) \Delta_{1} \zeta^{-\frac{\mu_{1}}{2}}+\Delta_{2} \zeta^{\frac{\mu_{1}}{2}}+O\left(\zeta^{1-\frac{\mu_{1}}{2}}\right)+O\left(\zeta^{1+\frac{\mu_{1}}{2}}\right) \\
f(0) & =\frac{\gamma_{1} \bar{V}_{1}}{1+\gamma_{1}}
\end{aligned}
$$

The explicit solution will lead to know exactly how the $\Delta_{i}$ coefficients are related to the fermionic constants of motion $\epsilon_{i}$ (see later on).

By using eq. (3.21) we can control all the divergences of the $X^{M}$ mapping in the interacting two-body problem, and also in the general $N$-body problem. Let us first describe the logarithmic cut in the $X^{a}$ variables, around particle $\# 1$ at $\zeta=0$. By inserting the development of the solution near the particle site, the $T$ and $Z$ variables contain logarithmic cuts 


$$
\begin{aligned}
& T \sim-i \int^{z} d z\left(\chi \psi^{\prime}+\psi \chi^{\prime}\right)+(\text { h.c. })=i \mu_{1} \Delta_{2} \Delta_{1}(1+f(0) \bar{f}(0)) \log \zeta+(h . c .) \\
& Z \sim-2 \int^{\zeta} d \zeta \psi \psi^{\prime}-2 \int^{\bar{\zeta}} d \zeta \overline{\chi \chi}^{\prime}=2 i \mu_{1} \Delta_{2} \Delta_{1} \bar{f}(0) \log \zeta-2 i \mu_{1} \bar{\Delta}_{1} \bar{\Delta}_{2} \bar{f}(0) \log \bar{\zeta} .
\end{aligned}
$$

Then we can conclude that the combination $Z-V_{1} T$ doesn't contain any logarithmic term around particle \#1 because :

$$
\frac{2 \bar{f}(0)}{1+f(0) \bar{f}(0)}=V_{1}
$$

This property will be very useful to discuss the bosonic part of the geodetic equations.

To discuss the fermionic geodesic equations, it is enough to develop the $\theta$ terms around particle \#1, obtaining that

$$
\begin{aligned}
& \sqrt{\frac{N}{f^{\prime}}} \simeq C \xi^{-\frac{\mathcal{M}}{4 \pi}} \zeta^{-\frac{\mu_{1}}{2}}\left(1+\bar{f}(0) \widetilde{C} \zeta^{\mu_{1}}\right)+O\left(\zeta^{1-\frac{\mu_{1}}{2}}\right)+O\left(\zeta^{1+\frac{\mu_{1}}{2}}\right) \\
& \sqrt{\frac{N}{f^{\prime}}} f \simeq C \xi^{-\frac{M}{4 \pi}} \zeta^{-\frac{\mu_{1}}{2}}\left(f(0)+\widetilde{C} \zeta^{\mu_{1}}\right)+O\left(\zeta^{1-\frac{\mu_{1}}{2}}\right)+O\left(\zeta^{1+\frac{\mu_{1}}{2}}\right) .
\end{aligned}
$$

Having fixed the singular behaviour of the fields, it is rather clear how to compute the fermionic geodesic. For example, from the $\Theta$-mapping we find that

$$
\Theta \sim \zeta^{-\frac{\mu_{1}}{2}}\left[\Delta_{1}+\theta C \xi^{-\frac{\mathcal{M}}{4 \pi}}\right]+i \bar{f}(0) \bar{\zeta}^{-\frac{\mu_{1}}{2}}\left[\bar{\Delta}_{1}+\overline{\theta C \xi}^{-\frac{\mathcal{M}}{4 \pi}}\right]
$$

which is solved by

$$
\theta(0)=\xi_{1}^{F}=-\frac{\Delta_{1}}{C} \xi^{\frac{\mathcal{M}}{4 \pi}} .
$$

For the $Z$-mapping and $T$-mapping we find:

$$
\begin{aligned}
& Z \sim-2 \psi(0) \zeta^{-\frac{\mu_{1}}{2}}\left[\Delta_{1}+\theta C \xi^{-\frac{\mathcal{M}}{4 \pi}}\right]-2 i \bar{f}(0) \bar{\chi}(0)\left[\bar{\Delta}_{1}+\overline{\theta C \xi}^{-\frac{\mathcal{M}}{4 \pi}}\right] \bar{\zeta}^{-\frac{\mu_{1}}{2}} \\
& T \sim-(i \chi(0)+\psi(0) f(0))\left[\Delta_{1}+\theta C \xi^{-\frac{\mathcal{M}}{4 \pi}}\right] \zeta^{-\frac{\mu_{1}}{2}}+(\text { h.c. })
\end{aligned}
$$

and the additional constraint 


$$
\left.\theta(0) \psi\right|_{\text {sing }}=\left.\theta(0) \chi\right|_{\text {sing }}=0,
$$

where $\left.\psi\right|_{\text {sing }}$ denotes the singular part of $\psi$ around the superparticle site. All of them are solved by a single equation (3.26). Therefore we have a non-perturbative proof that the cancellation of divergences is valid at all orders.

We are ready to describe the non-perturbative solution. This will be built in terms of hypergeometric functions, as in the case of gravity. Let us define the following basis of the hypergeometric equation

$$
\begin{aligned}
& y_{+}(0)=\zeta^{\frac{\mu_{1}}{2}}(1-\zeta)^{-\frac{\mu_{2}}{2}} \widetilde{F}\left(a^{\prime}, b^{\prime}, c^{\prime} ; \zeta\right) \\
& y_{-}(0)=\zeta^{-\frac{\mu_{1}}{2}}(1-\zeta)^{-\frac{\mu_{2}}{2}} \widetilde{F}(a, b, c ; \zeta)
\end{aligned}
$$

where we use the notation

$$
\widetilde{F}(a, b, c ; \zeta)=\frac{\Gamma(a) \Gamma(b)}{\Gamma(c)} F(a, b, c ; \zeta) .
$$

To match, in the static limit, the $\psi$ function, the correct choice for the coefficients $a, b$, and $c$ turns out to be, as in the gravity case,

$$
\begin{aligned}
a & =\frac{1}{2}\left(\frac{\mathcal{M}}{2 \pi}-\mu_{1}-\mu_{2}\right) \\
b & =1-\frac{1}{2}\left(\frac{\mathcal{M}}{2 \pi}+\mu_{1}+\mu_{2}\right) \\
c & =1-\mu_{1},
\end{aligned}
$$

and $a^{\prime}=a-c+1=a\left(-\mu_{1}\right), b^{\prime}=b-c+1=b\left(-\mu_{1}\right), c^{\prime}=2-c=c\left(-\mu_{1}\right)$. The basis, defined by eqs. (3.29) and (3.31) is also solution of the Fuchsian eq. (2.33) ( apart from an overall redefinition of $y$ by power factors ), with $\mu_{\infty}=\frac{\mathcal{M}}{2 \pi}-1$.

In the case of supergravity it is needed another basis, which we call $\tilde{y}_{ \pm}(0)$, to match, in the static limit, the $\chi$ function. It is similar to the above one with all the masses reversed in sign and it is given by

$$
\begin{aligned}
& \tilde{y}_{+}(0)=\zeta^{-\frac{\mu_{1}}{2}}(1-\zeta)^{\frac{\mu_{2}}{2}} \widetilde{F}\left(\tilde{a}^{\prime}, \tilde{b}^{\prime}, \tilde{c}^{\prime} ; \zeta\right) \\
& \tilde{y}_{-}(0)=\zeta^{\frac{\mu_{1}}{2}}(1-\zeta)^{\frac{\mu_{2}}{2}} \widetilde{F}(\tilde{a}, \tilde{b}, \tilde{c} ; \zeta)
\end{aligned}
$$

and

$$
\begin{aligned}
\tilde{a} & =-\frac{1}{2}\left(\frac{\mathcal{M}}{2 \pi}-\mu_{1}-\mu_{2}\right) \\
\tilde{b} & =1+\frac{1}{2}\left(\frac{\mathcal{M}}{2 \pi}+\mu_{1}+\mu_{2}\right) \\
\tilde{c} & =1+\mu_{1} .
\end{aligned}
$$


This function is solution of the Fuchsian equation (2.33) with a different choice of the index at infinity, namely $\left|\mu_{\infty}\right|=1+\frac{\mathcal{M}}{2 \pi}$.

The non perturbative solution can be expressed as:

$$
\begin{aligned}
\psi^{\prime} & =\Omega_{1}(\zeta)\left(y_{-}(0)+\bar{f}(0) k_{1} y_{+}(0)\right)+i \Omega_{2}(\zeta)\left(\bar{f}(0) \tilde{y}_{-}(0)+k_{2} \tilde{y}_{+}(0)\right) \\
\chi^{\prime} & =-i \Omega_{1}(\zeta)\left(f(0) y_{-}(0)+k_{1} y_{+}(0)\right)+\Omega_{2}(\zeta)\left(\tilde{y}_{-}(0)+k_{2} f(0) \tilde{y}_{+}(0)\right)
\end{aligned}
$$

where the meromorphic functions $\Omega_{1}(\zeta)$ and $\Omega_{2}(\zeta)$ are defined as

$$
\begin{aligned}
& \Omega_{1}(\zeta)=\frac{\delta_{1}}{\zeta}+\frac{\sigma_{1}}{1-\zeta} \\
& \Omega_{2}(\zeta)=\frac{\delta_{2}}{\zeta}+\frac{\sigma_{2}}{1-\zeta}
\end{aligned}
$$

and $\delta_{i}, \sigma_{i}$ are necessary parameters to satisfy the translation part of the monodromies. Instead the coefficients $k_{1}$ and $k_{2}$ are determined by imposing the monodromy conditions for $\psi^{\prime}$ and $\chi^{\prime}$ around $\zeta=1$. Let us introduce new bases around $\zeta=1$ whose monodromy is diagonal turning around particle \# 2

$$
\begin{aligned}
& y_{+}(1)=\zeta^{-\frac{\mu_{1}}{2}}(1-\zeta)^{\frac{\mu_{2}}{2}} \widetilde{F}\left(a^{\prime}, b^{\prime}, a^{\prime}+b^{\prime}-c^{\prime}+1 ; 1-\zeta\right) \\
& y_{-}(1)=\zeta^{-\frac{\mu_{1}}{2}}(1-\zeta)^{-\frac{\mu_{2}}{2}} \widetilde{F}(a, b, a+b-c+1 ; 1-\zeta),
\end{aligned}
$$

where $(a, b, c)$ are defined as in eq. (3.31), and

$$
\begin{aligned}
& \tilde{y}_{+}(1)=\zeta^{\frac{\mu_{1}}{2}}(1-\zeta)^{-\frac{\mu_{2}}{2}} \widetilde{F}\left(\tilde{a^{\prime}}, \tilde{b^{\prime}}, \tilde{a^{\prime}}+\tilde{b^{\prime}}-\tilde{c}^{\prime}+1 ; 1-\zeta\right) \\
& \tilde{y}_{-}(1)=\zeta^{\frac{\mu_{1}}{2}}(1-\zeta)^{\frac{\mu_{2}}{2}} \widetilde{F}(\tilde{a}, \tilde{b}, \tilde{a}+\tilde{b}-\tilde{c}+1 ; 1-\zeta),
\end{aligned}
$$

with $(\tilde{a}, \tilde{b}, \tilde{c})$ given by the other choice $(3.33)$.

It is not difficult to realize that, by using the rules of analytic continuation of the hypergeometric functions, $y_{+}(0)$ and $y_{-}(0)$ can be expressed in the new basis as:

$$
\begin{aligned}
& y_{+}(0)=K\left(\frac{\sin \pi a \sin \pi b}{\sin \pi(c-a) \sin \pi(c-b)} y_{-}(1)-y_{+}(1)\right) \\
& y_{-}(0)=K\left(y_{-}(1)-y_{+}(1)\right) \quad K=\frac{\pi}{\sin \pi(c-a-b) \Gamma(c-a) \Gamma(c-b)},
\end{aligned}
$$

where, apart from the common factor $K$, it appears the characteristic factor:

$$
\frac{\sin \pi a \sin \pi b}{\sin \pi(c-a) \sin \pi(c-b)}=\frac{\gamma_{12}-1}{\gamma_{12}+1}
$$

The formula (3.39) is valid for both choices (3.31) and (3.33) of the parameters of the hypergeometric function and therefore for both $y_{ \pm}$and $\tilde{y}_{ \pm}$, while $K=K(a, b, c)$ has to be substituted with $\widetilde{K}=K(\tilde{a}, \tilde{b}, \tilde{c})$. 
By comparing the analytic continuation of the solution near $\zeta=0$ with the expected solution near $\zeta=1$

$$
\begin{aligned}
\psi^{\prime} & =\widetilde{\Omega}_{1}(\zeta)\left(y_{-}(1)+\bar{f}(1) k_{1}^{\prime} y_{+}(1)\right)+i \widetilde{\Omega}_{2}(\zeta)\left(\bar{f}(1) \tilde{y}_{-}(1)+k_{2}^{\prime} \tilde{y}_{+}(1)\right) \\
\chi^{\prime} & =-i \widetilde{\Omega}_{1}(\zeta)\left(f(1) y_{-}(1)+k_{1}^{\prime} y_{+}(1)\right)+\widetilde{\Omega}_{2}(\zeta)\left(\tilde{y}_{-}(1)+f(1) k_{2}{ }_{2} \tilde{y}_{+}(1)\right),
\end{aligned}
$$

we find constraints on $k_{i}$ and $k_{i}^{\prime}$ which, for collinear velocities, are solved by:

$$
\begin{aligned}
k_{1} & =\bar{k}_{2}=\frac{\gamma_{12} \bar{V}_{21}}{\gamma_{12}-1} \\
k_{1}^{\prime} & =\bar{k}_{2}=\frac{\gamma_{12} V_{12}}{\gamma_{12}-1} .
\end{aligned}
$$

Instead $\Omega_{1}(\zeta)$ and $\Omega_{2}(\zeta)$ are computable from the monodromy conditions for the integrated fields $\psi$ and $\chi$ as in eq. (3.13), which allows to determine the anticommuting parameters in terms of two independent ones.

The singular behaviour of the fields can be easily obtained around $\zeta=0$

$$
\begin{aligned}
\psi & =\Delta_{1} \zeta^{-\frac{\mu_{1}}{2}}+i \bar{f}(0) \Delta_{2} \zeta^{\frac{\mu_{1}}{2}}+O\left(\zeta^{1-\frac{\mu_{1}}{2}}\right)+O\left(\zeta^{1+\frac{\mu_{1}}{2}}\right) \\
\chi & =-i f(0) \Delta_{1} \zeta^{-\frac{\mu_{1}}{2}}+\Delta_{2} \zeta^{\frac{\mu_{1}}{2}}+O\left(\zeta^{1-\frac{\mu_{1}}{2}}\right)+O\left(\zeta^{1+\frac{\mu_{1}}{2}}\right) \\
\Delta_{1} & =-\frac{2}{\mu_{1}}\left[\delta_{1} \frac{\Gamma(a) \Gamma(b)}{\Gamma(c)}+i \delta_{2} k_{2} \frac{\Gamma\left(\tilde{a}^{\prime}\right) \Gamma\left(\tilde{b}^{\prime}\right)}{\Gamma\left(\tilde{c}^{\prime}\right)}\right] \\
\Delta_{2} & =\frac{2}{\mu_{1}}\left[\delta_{2} \frac{\Gamma(\tilde{a}) \Gamma(\tilde{b})}{\Gamma(\tilde{c})}-i \delta_{1} k_{1} \frac{\Gamma\left(a^{\prime}\right) \Gamma\left(b^{\prime}\right)}{\Gamma\left(c^{\prime}\right)}\right]
\end{aligned}
$$

and around $\zeta=1$

$$
\begin{aligned}
\psi & \sim \tilde{\Delta}_{1}(1-\zeta)^{-\frac{\mu_{2}}{2}}+i \bar{f}(1) \tilde{\Delta}_{2}(1-\zeta)^{\frac{\mu_{2}}{2}}+O\left((1-\zeta)^{1-\frac{\mu_{2}}{2}}\right)+O\left((1-\zeta)^{1+\frac{\mu_{2}}{2}}\right) \\
\chi & \sim-i f(1) \tilde{\Delta}_{1}(1-\zeta)^{-\frac{\mu_{2}}{2}}+\tilde{\Delta}_{2}(1-\zeta)^{\frac{\mu_{2}}{2}}+O\left((1-\zeta)^{1-\frac{\mu_{2}}{2}}\right)+O\left((1-\zeta)^{1+\frac{\mu_{2}}{2}}\right) \\
\tilde{\Delta}_{1} & =\frac{2}{\mu_{2}}\left[\sigma_{1} H \frac{\Gamma(a) \Gamma(b)}{\Gamma(a+b-c+1)}+i \sigma_{2} \widetilde{H} k^{\prime}{ }_{2} \frac{\Gamma\left(\tilde{a}^{\prime}\right) \Gamma\left(\tilde{b}^{\prime}\right)}{\Gamma\left(\tilde{a}^{\prime}+\tilde{b}^{\prime}-\tilde{c}^{\prime}+1\right)}\right] \\
\tilde{\Delta}_{2} & =\frac{2}{\mu_{2}}\left[-\sigma_{2} \widetilde{H} \frac{\Gamma(\tilde{a}) \Gamma(\tilde{b})}{\Gamma(\tilde{a}+\tilde{b}-\tilde{c}+1)}+i \sigma_{1} H k^{\prime} \frac{\Gamma\left(a^{\prime}\right) \Gamma\left(b^{\prime}\right)}{\Gamma\left(a^{\prime}+b^{\prime}-c^{\prime}+1\right)}\right] \\
H & =K\left(1+\bar{f}(0) \frac{\gamma_{12} \bar{V}_{12}}{\gamma_{12}+1}\right) \quad \widetilde{H}=\widetilde{K}\left(1+f(0) \frac{\gamma_{12} V_{21}}{\gamma_{12}+1}\right) .
\end{aligned}
$$

The logarithmic behaviour of $T$ and $Z$ variables is constrained by the property that $Z-V_{i} T$ are free of singularities, and therefore it is enough to compute the one for $T$ around each particle

$$
\begin{array}{lll}
\Delta_{S} T & \stackrel{1}{\rightarrow} & \Delta_{S} T+4 \pi \mu_{1} \operatorname{Re}\left(\Delta_{1} \Delta_{2}\right)(1+f(0) \bar{f}(0)) \\
\Delta_{S} T & \stackrel{2}{\rightarrow} & \Delta_{S} T+4 \pi \mu_{2} \operatorname{Re}\left(\widetilde{\Delta}_{1} \widetilde{\Delta}_{2}\right)(1+f(1) \bar{f}(1)) .
\end{array}
$$


By solving the system of conditions we find that $\Omega_{1}(\zeta)$ and $\Omega_{2}(\zeta)$ are of order $O\left(V^{2}\right)$. Then the static limit is recovered because $y_{-}(0)$ and $\tilde{y}_{-}(0)$ scale as $O\left(1 / V^{2}\right)$ due to the vanishing of the $a$ parameter in the hypergeometric function $\widetilde{F}(a, b, c, \zeta)$.

By putting together the solution for gravity, described in Sec. 2, and the exact solution for the gravitino fields, given in eq. (3.34), we have complete control of the fields. Moreover the fermionic trajectory is already determined in terms of the bosonic one by eq. (3.26):

$$
\theta(0)=-\frac{\Delta_{1}}{C} \xi^{\frac{\mathcal{M}}{4 \pi}} \quad \theta(1)=-\frac{\widetilde{\Delta}_{1}}{C} \xi^{\frac{\mathcal{M}}{4 \pi}} .
$$

Let us note that the difference of fermionic coordinates in this case may depend on the residual arbitrariness of the anticommuting parameters which persists after solving the monodromy conditions. However, it is possible to add homogeneous solutions of the monodromies, depending on arbitrary parameters $\alpha_{i}=\alpha_{i}\left(\mu_{j}, \mathcal{M}\right), \beta_{i}=\beta_{i}\left(\mu_{j}, \mathcal{M}\right)$, to the gravitino fields

$$
\begin{aligned}
\tilde{\psi} & =\psi+\Delta \psi \quad \Delta \psi \rightarrow \bar{a}_{i} \Delta \psi+i \bar{b}_{i} \Delta \chi \\
\Delta \psi & =\left(\alpha_{1} \delta_{1}+\beta_{1} \sigma_{1}\right)\left[y_{-}(0)+\bar{f}(0) k_{1} y_{+}(0)\right]+i\left(\alpha_{2} \delta_{2}+\beta_{2} \sigma_{2}\right)\left[\bar{f}(0) \tilde{y}_{-}(0)+k_{2} \tilde{y}_{+}(0)\right] \\
\tilde{\chi} & =\chi+\Delta \chi \quad \Delta \chi \rightarrow a_{i} \Delta \chi-i b_{i} \Delta \psi \\
\Delta \chi & =-i\left(\alpha_{1} \delta_{1}+\beta_{1} \sigma_{1}\right)\left[f(0) y_{-}(0)+k_{1} y_{+}(0)\right]+\left(\alpha_{2} \delta_{2}+\beta_{2} \sigma_{2}\right)\left[\tilde{y}_{-}(0)+k_{2} f(0) \tilde{y}_{+}(0)\right]
\end{aligned}
$$

which do not change the good properties of the solution but instead are able to connect $\theta(1)-\theta(0)$ only to a linear combination of the constants of motion.

It remains to be investigated the equation for $\xi(t)$ which solves the whole motion of superparticles. At level of the bosonic trajectory

$$
B_{2}-B_{1}=Z_{2}-Z_{1}-V_{2} T_{2}+V_{1} T_{1}
$$

we expect to find finite contributions derived from the $\xi_{i}^{F}$ fermionic trajectory. Let us start to analyze the $T$-mapping. In this case the finite term due, for example, to $\xi_{1}^{F}$ is given, in the normalization of eq. (3.21) by the term

$$
\left.\theta(i \chi+\psi f) \sqrt{\frac{N}{f^{\prime}}}\right|_{0}=i(1+\bar{f}(0) f(0)) \Delta_{2} \Delta_{1}+(\text { h.c. }),
$$

which is obtained by a compensation of $\xi^{ \pm \mu_{i}}$ exponents.

In the $Z$-mapping there is also a finite contribution due to $\xi_{1}^{F}$ which might enter into the geodesic equations 3.47 


$$
\left.2 \theta \psi \sqrt{\frac{N}{f^{\prime}}}\right|_{0}-\left.2 i \bar{\chi} \sqrt{\frac{\bar{N}}{\bar{f}}} \overline{f \theta}\right|_{0}=2 \bar{f}(0)\left(i \Delta_{2} \Delta_{1}+(\text { h.c. })\right) .
$$

It is interesting to observe that, by taking the combination $Z\left(\xi_{i}\right)-V_{i} T\left(\xi_{i}\right)$, these finite contributions cancel exactly due again to eq. (3.23) and from now on we can limit our discussion to the $\theta=0$ part of eq. (3.47).

However there is another obstacle to consider, namely the logarithmic divergences which make the geodetic equations (3.47) undetermined. To define them properly avoiding the logarithmic ambiguities, it is enough to compare the two superparticles from a fixed reference point $C$. For simplicity we take $C$ in a fixed position in the $\zeta$-plane, since then it is easy to compare the $\xi$-dependence of the various terms which contribute to the geodesics.

Since the combinations $Z\left(\xi_{i}\right)-V_{i} T\left(\xi_{i}\right)$ are well defined, it is convenient to compute the following building block of the geodesics equations ( without the $\theta$ contributions ):

$$
\begin{aligned}
\left(Z\left(\xi_{i}\right)-\right. & \left.Z(C)-V_{i} T\left(\xi_{i}\right)+V_{i} T(C)\right)\left.\right|_{\theta=0}=\int_{C}^{\xi_{i}}\left(\frac{N}{f^{\prime}}-2 \psi \psi^{\prime}\right) d z+ \\
+ & \int_{\bar{C}}^{\bar{\xi}_{i}}\left(\frac{\bar{N}}{\bar{f}^{\prime}} \bar{f}-2 \overline{\chi \chi}^{\prime}\right) d \bar{z}-V_{i}\left[\int_{C}^{\xi_{i}} d z\left(\frac{N f}{f^{\prime}}-i\left(\chi \psi^{\prime}+\psi \chi^{\prime}\right)+(\text { h.c. })\right]\right. \\
& (i=1,2) .
\end{aligned}
$$

Since $\psi(x)$ depends only on $\zeta$, all the fermionic integrals scale as a constant $\left(\xi^{0}\right)$, while the bosonic integral scales as $\xi^{1-\frac{\mathcal{M}}{2 \pi}}$. Therefore, for large times, the pure bosonic contribution dominates ( the gravitino has shorter range than the graviton ). By taking the difference of the building blocks of eq. (3.50) we obtain a regularized version of eq. (3.47)

$$
\begin{aligned}
Z\left(\xi_{2}\right) & -V_{2} T\left(\xi_{2}\right)-Z\left(\xi_{1}\right)+V_{1} T\left(\xi_{1}\right)=B\left(\xi_{2}\right)-B\left(\xi_{1}\right)= \\
& =\left(V_{1}-V_{2}\right) T(C)+\int_{\xi_{1}}^{\xi_{2}}\left(\frac{N}{f^{\prime}}-2 \psi \psi^{\prime}\right) d z+\int_{\bar{\xi}_{1}}^{\bar{\xi}_{2}}\left(\frac{\bar{N}}{\bar{f}^{\prime}} \bar{f}-2 \overline{\chi \chi}\right) d \bar{z} \\
& -V_{2}\left[\int_{C}^{\xi_{2}} d z\left(\frac{N f}{f^{\prime}}-i\left(\chi \psi^{\prime}+\psi \chi^{\prime}\right)+(\text { h.c. })\right]+\right. \\
& +V_{1}\left[\int_{C}^{\xi_{1}} d z\left(\frac{N f}{f^{\prime}}-i\left(\chi \psi^{\prime}+\psi \chi^{\prime}\right)+(\text { h.c. })\right] .\right.
\end{aligned}
$$

We have eliminated $Z(C)$, and we are left with $T(C)$ which is the freedom of choosing an universal time, up to time reparametrizations. For large time $T(C) \sim t$, while it is clear that the dominant term, in the remaining part, is given by the pure bosonic contribution. Therefore it is possible to define a scattering angle at the classical level and it coincides with the result of the gravity case [7]. This was also expected at the level of the monodromy 
matrices $L_{i}$, since the scattering angle is strictly related to the invariant mass of the system which is computable from the Lorentz part of the composite monodromy $L_{12}=L_{1} L_{2}$, while the supersymmetric cut gives contribution only to the translation part.

The main effect of the anticommuting variables is to introduce a certain level of unpredictability of the trajectory, but not of the scattering angle. To introduce probabilities for the scattering angle we need quantum mechanics [3].

\subsection{Extensions}

A natural extension would be to apply this method to the study of the $N$ body problem. We have already investigated it in the case of point particles in $(2+1)$ gravity. In that case the solution is found by requiring that the function $f(z, t)$ respects the projective transformations (2.32) around each particle. Since the monodromy matrices are constants of motion, we have to deal with an isomonodromy problem [21], which is solved at level of $f$ with the introduction of apparent singularities in a Fuchsian differential equation analogous to (2.33). The isomonodromy then leads to constraint the motion of apparent singularities in terms of the physical ones. Therefore since the monodromy problem for $\psi$ and $\chi$ is very similar to the one for $f(z, t)$ one expects that apparent singularities must be present in their solution too. From the two-body problem we can deduce that their general solution can be represented as a sum of two distinct solutions of a Fuchsian differential equation, having $(N+1)$ physical poles and $N-2$ apparent singularities. The two solutions are different because all masses enter with opposite sign, and in particular this is also true for the total mass of the system $\mathcal{M}$ related to the loop invariant $L_{1} L_{2} \ldots L_{N}$. Therefore in one case the index at infinity has to be defined as $\mu_{\infty}=\frac{\mathcal{M}}{2 \pi}-1$, in the other case as $\left|\mu_{\infty}\right|=\frac{\mathcal{M}}{2 \pi}+1$.

We will not proceed further in this direction. In any case, it seems to us obvious that a solution to the monodromy problem (2.36) must exists for $N$ superparticles. Instead we would like to investigate the static case for three bodies $\left(z=\xi_{i}, i=1,2,3\right)$, in order to clarify the procedure for obtaining the static solution, which gives an idea of the parameterization of the interacting solution. In the static limit the $\psi$ function can be expressed, by choosing an adimensional variable $\zeta=\left(z-\xi_{1}\right) /\left(\xi_{2}-\xi_{1}\right)$ and $\xi=\xi_{13} / \xi_{12}$, as follows

$$
\psi^{\prime}(\zeta)=\left(\frac{\delta_{1}}{\zeta}+\frac{\sigma_{1}}{\zeta-1}+\frac{\rho_{1}}{\zeta-\xi}\right) \zeta^{-\frac{\mu_{1}}{2}}(\zeta-1)^{-\frac{\mu_{2}}{2}}(\zeta-\xi)^{-\frac{\mu_{3}}{2}}
$$

Around the superparticles the singular behaviour of the $\Theta$-mapping, at the level of $\theta$-terms, 
constraints the fermionic geodetics to be:

$$
\theta(0)=-\frac{2}{\mu_{1}} \delta_{1} \xi_{12}^{\frac{\mu_{1}+\mu_{2}+\mu_{3}}{2}} \quad \theta(1)=-\frac{2}{\mu_{2}} \sigma_{1} \xi_{12}^{\frac{\mu_{1}+\mu_{2}+\mu_{3}}{2}} \quad \theta(\xi)=-\frac{2}{\mu_{3}} \rho_{1} \xi_{12}^{\frac{\mu_{1}+\mu_{2}+\mu_{3}}{2}} .
$$

Analogously, $\chi^{\prime}(\zeta)$ can be defined as:

$$
\chi^{\prime}(\zeta)=\left(\frac{\delta_{2}}{\zeta}+\frac{\sigma_{2}}{\zeta-1}+\frac{\rho_{2}}{\zeta-\xi}\right) \zeta^{\frac{\mu_{1}}{2}}(\zeta-1)^{\frac{\mu_{2}}{2}}(\zeta-\xi)^{\frac{\mu_{3}}{2}}
$$

Imposing the monodromy conditions

$$
\begin{array}{ll}
\psi(\xi)-\psi(0)=\int_{0}^{\xi} d \zeta \psi^{\prime} & \chi(\xi)-\chi(0)=\int_{0}^{\xi} d \zeta \chi^{\prime} \\
\psi(1)-\psi(0)=\int_{0}^{1} d \zeta \psi^{\prime} & \chi(1)-\chi(0)=\int_{0}^{1} d \zeta \chi^{\prime} .
\end{array}
$$

allows to compute four anticommuting unknowns in terms of two independent ones. Moreover the differences of coordinates $\theta\left(\xi_{i}\right)-\theta\left(\xi_{j}\right)$ are always dependent only on the constant of motions, as in the two-body static case. In general, for a static $N$ body problem, formulas similar to eqs. (3.52) and (3.54) hold carrying $N$ anticommuting parameters.

Finally, we expect that solving the $N$-body problem in supergravity is no more difficult than in gravity, where, although the non perturbative $N$-body solution cannot be given in terms of known tabulated functions, it is possible to construct solutions to the monodromy matrices, at least perturbatively in $V_{i}$ []].

Another straightforward extension of this formalism is to understand extended supergravity. For example, $N=2$ supersymmetry has two $\Theta_{i}$, superspace variables, and the corresponding supermetric is

$$
\begin{aligned}
d s^{2} & =\left[d T+\frac{i}{2}\left(\Theta_{i} d \bar{\Theta}_{i}+\bar{\Theta}_{i} d \Theta_{i}\right)\right]^{2}- \\
& -\left(d Z+\Theta_{i} d \Theta_{i}\right)\left(d \bar{Z}+d \bar{\Theta}_{i} \bar{\Theta}_{i}\right)-\left(d \Theta_{i} d \bar{\Theta}_{i}\right) .
\end{aligned}
$$

Besides the supersymmetric translation cuts for $\Theta_{i}$, it is also possible to have cuts related to the mixing of the two $\Theta_{i}$ which however must leave the bilinears $\Theta_{i} d \Theta_{i}$, and $\Theta_{i} d \bar{\Theta}_{i}+\bar{\Theta}_{i} d \Theta_{i}$ invariant. In any case, the bosonic variables $(T, Z)$ feel only the supersymmetric cuts $\Theta_{i} \rightarrow$

$\Theta_{i}+\epsilon_{i}$. Straightforward generalizations of our parameterization (2.37) allow to solve any kind of supersymmetric $N$-body problem always in terms of analytic functions.

\section{Conclusion}

In this paper we have been able to solve explicitly the non-perturbative interaction between $N$ superparticles and supergravity. We have analyzed in detail the two-superbody system in 
our coordinates with instantaneous propagation, and sketched the classical $N$-body problem. Our method consists of the following steps. Firstly we have introduced a new parameterization for the $X$-supermapping, which extends the well known superanalytic mapping, and gives the general solution to the equations of motion of pure supergravity in terms of arbitrary analytic functions.

Introducing specific polydromies in the mapping we can couple in a consistent way $N$ superparticles to $(2+1)$ supergravity. The bootstrap between motion of superparticles and field equations is achieved by imposing constraints which are equivalent to integrate the supergeodetic equations. In addition to evaluate the bosonic distance of the superparticles, a criterion which was known from the gravity problem, we have found, by examining in detail the explicit solution of the supermapping, a second fermionic constraint. It represents the odd part of the supergeodesic equations and gives a precise meaning to a non perturbative divergence at the particle site, which was already present in the case of spinning particles coupled to gravity.

The requirement of cancellation of the fermionic residue at this divergence is found to be consistent at all orders, by using only general properties of the monodromy matrices. This second constraint is an interesting outcome of this work, which was difficult to carry out because of the lack of explicit examples available with anticommuting variables.

Finally, we have learned that divergences cannot be considered negligible, as we usually do in field theory, but have a important role to build a more complete theory.

\section{Acknowledgements}

I would like to thank Dr. A. Bellini and Prof. M. Ciafaloni for useful discussions.

\section{A Appendix - Two-body solution in $(2+1)$-gravity}

In this Appendix we review the solution of $(2+1)$-gravity. To build a representation of the matching conditions around the particles, i.e. to impose that for $\left(z-\xi_{i}\right) \rightarrow e^{2 \pi i}\left(z-\xi_{i}\right)$

$$
d X^{a} \rightarrow\left(L_{i}\right)_{b}^{a} d X^{b}
$$

we introduced the analytic function $f(z, t)$, which has branch points at $z=\xi_{i}(t)$ such that, when $z$ turns around $\xi_{i}, f$ transforms as a projective representation of the monodromies

$$
f(z, t) \rightarrow \frac{a_{i} f(z, t)+b_{i}}{\bar{b}_{i} f(z, t)+\bar{a}_{i}}, \quad(i=1, \ldots, N)
$$


with

$$
\begin{gathered}
a_{i}=\cos \frac{m_{i}}{2}+i \gamma_{i} \sin \frac{m_{i}}{2}, \quad b_{i}=-i \gamma_{i} \bar{V}_{i} \sin \frac{m_{i}}{2} \\
\gamma_{i} \equiv\left(1-\left|V_{i}\right|^{2}\right)^{-1 / 2}, \quad V_{i}=\left(P_{i}^{x}+i P_{i}^{y}\right) / E_{i} .
\end{gathered}
$$

The specific form of $f(\zeta, t)$ for the two-body problem is given by

$$
f(\zeta, t)=\frac{f(0)+f_{(1)}}{1+\bar{f}(0) f_{(1)}} .
$$

The value $f(0)$ is determined by the $L_{1}$ monodromy matrix

$$
f(0)=\frac{\gamma_{1} \bar{V}_{1}}{1+\gamma_{1}}
$$

and it corresponds to the fixed point of the projective transformation (A.2). $f_{(1)}$ would be the solution for the monodromies (A.2) if we decide to see the particle scattering in the particle \#1 rest frame:

$$
f_{(1)}(\zeta)=\frac{\gamma_{12} \bar{V}_{21}}{\gamma_{12}-1} \frac{\zeta^{\mu_{1}} \widetilde{F}\left(a^{\prime}, b^{\prime}, c^{\prime} ; \zeta\right)}{\widetilde{F}(a, b, c ; \zeta)} .
$$

In fact, the monodromy around particle $\# 1$ becomes a pure rotation . To derive this formula we have assumed for simplicity that the velocities are collinear, since then the relativistic difference of velocities simplifies to

$$
V_{12}=\frac{V_{1}-V_{2}}{1-\bar{V}_{1} V_{2}}
$$

and $\gamma_{12} \equiv\left(P_{1} P_{2}\right) / m_{1} m_{2}$ is the relative $\gamma$-factor. The factor in front to the ratio of the hypergeometric functions is chosen to satisfy the monodromy conditions around particle \#2, and in particular to obtain the value

$$
f(\zeta=1)=\frac{\gamma_{2} \bar{V}_{2}}{1+\gamma_{2}} .
$$

In the formula (A.6) we have introduced a modified hypergeometric function solution of the hypergeometric equation (2.33)

$$
\widetilde{F}(a, b, c ; z) \equiv \frac{\Gamma(a) \Gamma(b)}{\Gamma(c)} F(a, b, c ; z) .
$$

The choice of the parameteres of the hypergeometric function is defined as follows:

$$
\begin{aligned}
a\left(\mu_{1}\right) & =\frac{1}{2}\left(1+\mu_{\infty}-\mu_{1}-\mu_{2}\right), \quad b\left(\mu_{1}\right)=\frac{1}{2}\left(1-\mu_{\infty}-\mu_{1}-\mu_{2}\right), c\left(\mu_{1}\right)=1-\mu_{1} \\
a^{\prime} & =a\left(-\mu_{1}\right), b^{\prime}=b\left(-\mu_{1}\right), c^{\prime}=c\left(-\mu_{1}\right) .
\end{aligned}
$$


The values of the indices $\mu_{i}$ are defined in eq. (2.34).

The two hypergeometric function have a natural definition of their cuts which entails the structure of cuts for $f(z, t)$, which split the $z$-plane in two half-planes, apart from a segment of continuity on the real axis between 0 and 1 . In the mass range

$$
0 \leq m_{1}, m_{2}, \mathcal{M} \leq 2 \pi
$$

the upper half $z$-plane is mapped on a triangle whose edges are circular arcs, and whose internal angles are $\frac{m_{1}}{2}, \frac{m_{2}}{2}$ and $\pi-\frac{\mathcal{M}}{2}$, for $m_{1}+m_{2} \leq \mathcal{M} \leq 2 \pi$. The lower half plane is obtained by Schwarz's reflection of this triangle. The whole $z$-plane is thus mapped to a region inside the $f$ unit disk, $|f(z, t)| \leq 1$, and the same inequality is satisfied by $f(z, t)$ on any other Riemann sheet.

In particular $f(z, t)$ at $\infty$ has two values, one for each half plane, $f_{+}(\infty)\left(f_{-}(\infty)\right)$, which are fixed points of the composite loop operators $L_{21}\left(L_{12}\right)$. Until the constant velocities do not saturate the Carlip bound $\cos \frac{\mathcal{M}}{2}<-1$, the value of $f_{ \pm}(\infty)$ is always contained into the $f$-unit disk and the determinant of the metric is always non vanishing. This property extends to the supergravity case, since the anticommuting parameters do not play a significant role in inverting the supermetric, until there is a non vanishing pure bosonic part.

\section{References}

[1] A. Staruszkiewicz, Acta Phys. Polonica 24 (1963) 735.

[2] S. Deser, R. Jackiw and G. 't Hooft, Ann. Phys. (N.Y.) 152 (1984) 220.

[3] G. 't Hooft, Comm. Math. Phys. 117 (1988) 685; S. Deser and R. Jackiw, Comm. Math. Phys. 118 (1988) 495.

[4] J.R. Gott and M. Alpert, Gen. Relativ. Gravitation 16 (1984) 243; J.R. Gott, Astrophys. J. 288 (1985) 422.

[5] A. Cappelli, M. Ciafaloni and P. Valtancoli, Nucl. Phys. B369 (1992) 669; Phys. Lett. B273 (1991) 431.

[6] A. Bellini and P. Valtancoli, Phys. Lett. B348(1995) 44; A. Bellini, M. Ciafaloni and P. Valtancoli, Nucl. Phys. B454 (1995) 449.

[7] A. Bellini, M. Ciafaloni and P. Valtancoli, Phys. Lett. B357 (1995) 532 ; A. Bellini, M. Ciafaloni and P. Valtancoli, Nucl. Phys. B462 (1996) 453. 
[8] M. Welling, Class. and Quant. Grav. 13 (1996), 653.

[9] K. Koehler, F. Mansouri, Cenalo Vaz, L. Witten, Nucl. Phys. B358 (1991), 677-694; J. Math. Phys. 32 (1991), 239-246; Nucl. Phys. B341 (1990), 167-186.

[10] G. Clement, Int. J. Theor. Phys. 24 (1985) 267; P. de Sousa Gerbert and R. Jackiw, Comm. Math. Phys. 124 (1989) 229; P. de Sousa Gerbert, Nucl. Phys. B 346 (1990) 440.

[11] M. Ciafaloni, P. Valtancoli, Class. Quant. Grav. 14 955, 1997.

[12] J. R. Gott, Phys. Rev. Lett. 66 (1991) 1126; S. Deser, R. Jackiw and 't Hooft, Phys. Rev. Lett. 68 (1992) 267; P. Menotti and D. Seminara, Phys. Lett. B301 (1992) 25.

[13] S.J. Gates, M.T. Grisaru, M. Rocek, W. Siegel, "Superspace or one thousand and one lessons in supersymmetry", New York Benjamin/cummings ( 1983) ( Frontiers In Physics, 58);

[14] F.A. Berezin, M.S. Marinov, Annals Phys. 104 (1977), 336.

[15] J. Bagger, J. Wess, "Supersymmetry and Supergravity", Princeton University Press (1990);

[16] B. DeWitt, "Supermanifolds", Cambridge Monographs on Mathematical Physics, Cambridge University Press (1984).

[17] A. Rogers, J.Math.Phys. 21; 1352,1980.

[18] E. Witten, Nucl. Phys. B311 (1988) 46, B323 (1989) 113; A. Achucarro and P. K. Townsend, Phys. Lett. B180 (1986) 89, B229 (1989) 383.

[19] R. Arnowitt, S. Deser and C.W. Misner, in L. Witten (ed.) "Gravitation: an introduction to current research", Wiley, New York (1962); B. S. DeWitt, Phys. Rev. 160 (1967) 1113.

[20] S. Carlip, Nucl. Phys. B324 (1989) 106.

[21] M. Yoshida "Fuchsian Differential equations", Max-Planck-Institut für Mathematik, Bonn (1987), Fried. Vieweg \& Sohn Editor.; R. Fuchs, Mat. Annalen 63 (1907), 301; R. Garnier, Ann. Sci. Ecole Norm. Sup. (3) 29 (1912), 1. 